\title{
Proving the equivalence of $c$-extremization and its gravitational dual for all toric quivers
}

\author{
Seyed Morteza Hosseini ${ }^{a}$ and Alberto Zaffaroni ${ }^{b, c}$ \\ ${ }^{a}$ Kavli IPMU (WPI), UTIAS, The University of Tokyo, \\ Kashiwa, Chiba 277-8583, Japan \\ ${ }^{b}$ Dipartimento di Fisica, Università di Milano-Bicocca, \\ I-20126 Milano, Italy \\ ${ }^{c}$ INFN, sezione di Milano-Bicocca, \\ I-20126 Milano, Italy \\ E-mail: morteza.hosseini@ipmu.jp, alberto.zaffaroni@mib.infn.it
}

ABSTRACT: The gravitational dual of $c$-extremization for a class of $(0,2)$ two-dimensional theories obtained by twisted compactifications of D3-brane gauge theories living at a toric Calabi-Yau three-fold has been recently proposed. The equivalence of this construction with $c$-extremization has been checked in various examples and holds also off-shell. In this note we prove that such equivalence holds for an arbitrary toric Calabi-Yau. We do it by generalizing the proof of the equivalence between $a$-maximization and volume minimization for four-dimensional toric quivers. By an explicit parameterization of the R-charges we map the trial right-moving central charge $c_{r}$ into the off-shell functional to be extremized in gravity. We also observe that the similar construction for M2-branes on $\mathbb{C}^{4}$ is equivalent to the $\mathcal{I}$-extremization principle that leads to the microscopic counting for the entropy of magnetically charged black holes in $\mathrm{AdS}_{4} \times S^{7}$. Also this equivalence holds off-shell.

Keywords: AdS-CFT Correspondence, Black Holes in String Theory, Supersymmetric Gauge Theory

ARXIV EPRINT: 1901.05977 


\section{Contents}

1 Introduction 1

2 Introducing the field theory $\quad 4$

$2.1 \mathcal{N}=1$ superconformal field theories 4

$\begin{array}{lll}2.2 & \text { Twisted compactification to two dimensions } & 7\end{array}$

3 c-extremization equals its gravity dual for all toric quivers $\quad 8$

$3.1 a$-maximization is volume minimization 8

$3.2 c$-extremization is equivalent to its gravity dual 9

4 Formulae for the R-charges and examples $\quad 12$

$\begin{array}{ll}4.1 \mathcal{N}=4 \mathrm{SYM} & 13\end{array}$

$\begin{array}{lll}4.2 & \text { Klebanov-Witten theory } & 14\end{array}$

$\begin{array}{lll}4.3 Y^{p, q} & \text { quiver gauge theory } & 16\end{array}$

5 I-extremization and black hole entropy $\quad 17$

$\begin{array}{lll}5.1 & \text { The ABJM theory } & 18\end{array}$

A The relation between $c_{r}\left(\Delta_{a}, \mathfrak{n}_{a}\right)$ and $a\left(\Delta_{a}\right) \quad 21$

$\begin{array}{ll}\text { A.1 Direct evaluation } & 21\end{array}$

$\begin{array}{lll}\text { A.2 Integrating the anomaly polynomial } & 22\end{array}$

B Proof of the equality between $c\left(b_{i}, \mathfrak{n}_{a}\right)$ and $c_{r}\left(\Delta_{a}, \mathfrak{n}_{a}\right) \quad 23$

B.1 Some simplifications 23

$\begin{array}{lll}\text { B.2 A convenient gauge } & 24\end{array}$

$\begin{array}{ll}\text { B.3 Completing the proof } & 26\end{array}$

B.4 Proving that $\mathcal{V}_{\text {on-shell }}\left(b_{i}, \mathfrak{n}_{a}\right)=a\left(\Delta_{a}\right) \quad 30$

\section{Introduction}

Central charges play an important role in the study of superconformal field theories (SCFTs) in even dimensions. In supersymmetric gauge theories the R-symmetry current is not necessarily unique and mixes with the global symmetry currents. This happens in particular in most models with a holographic dual. It is well known that, for $\mathcal{N}=1$ supersymmetric theories in four dimensions, the extremization of a trial central charge $a$ with respect to a varying R-symmetry allows to identify the exact R-symmetry of the superconformal theory [1]. ${ }^{1}$ Similarly, for $\mathcal{N}=(0,2)$ supersymmetric theories in two dimensions, the extremization of a right-moving trial central charge $c_{r}$ allows to identify the

\footnotetext{
${ }^{1}$ The exact R-symmetry is the one appearing in the superconformal algebra.
} 
exact R-symmetry $[2,3]$. The gravity dual of $a$-maximization is the volume minimization principle discovered in $[4,5] .{ }^{2}$ The equivalence of $a$-maximization and volume minimization has been proven in [9] for all quivers associated with D3-branes at toric Calabi-Yau three-fold singularities and generalized in $[10,11]$. On the other hand, the gravity dual of $c$-extremization has been recently found in a series of very interesting papers $[12,13]$. The authors of $[12,13]$ have checked the equivalence of their formalism with $c$-extremization in various explicit examples. It is the purpose of this note to prove this equivalence for all theories obtained by twisted compactifications of D3-branes sitting at an arbitrary toric Calabi-Yau three-fold, just by generalizing the arguments of [9].

The main focus in this note are theories that are obtained by a twisted compactification of four-dimensional $\mathcal{N}=1$ superconformal theories living on D3-branes sitting at the tip of a toric Calabi-Yau cone $C\left(Y_{5}\right)$ over a Sasaki-Einstein manifold $Y_{5}$. These four-dimensional theories are well known and classified in terms of the toric data [14-16]. The gravitational dual is $\mathrm{AdS}_{5} \times Y_{5}$. When compactified on a Riemann surface $\Sigma_{\mathfrak{g}}$ with a topological twist parameterized by magnetic fluxes $\mathfrak{n}_{a}$, the theory can flow in the infrared (IR) to a $\mathcal{N}=(0,2)$ CFT. The gravity solution dual to such CFT is a warped background $\mathrm{AdS}_{3} \times_{W} Y_{7}$, where $Y_{7}$ is topologically a fibration of $Y_{5}$ over $\Sigma_{\mathfrak{g}}$, with a five-form flux.

Given the close similarity between the gravitational dual of $a$ - and $c$-extremization, let us start by first reviewing the story for $a$-maximization in four-dimensions. By relaxing the equations of motion but still imposing the conditions for supersymmetry, the authors of $[4,5]$ defined an off-shell class of supersymmetric backgrounds obtained by replacing $Y_{5}$ with a general Sasaki manifold. The background depends on a Reeb vector, $b=\left(b_{1}, b_{2}, b_{3}\right)$, which specifies the direction of the R-symmetry inside the three isometries of $Y_{5}$. It has been shown in $[4,5]$ that the extremization of the volume of the Sasaki manifold identifies the exact R-symmetry of the CFT and allows to compute its central charge. The proof that this procedure is equivalent to $a$-maximization involves choosing a convenient parameterization of the R-charges of the toric quiver in terms of the toric data and define a natural parameterization of the R-charges in terms of the Reeb vector [9]

$$
\Delta_{a}\left(b_{i}\right)=\frac{\pi \operatorname{Vol}\left(S_{a}\left(b_{i}\right)\right)}{b_{1} \operatorname{Vol}\left(Y_{5}\left(b_{i}\right)\right)}
$$

where $S_{a}$ are toric three-cycles in $Y_{5}$. One then shows that

$$
\left.\frac{\pi^{3} N^{2}}{4 \operatorname{Vol}\left(Y_{5}\left(b_{i}\right)\right)} \equiv a\left(\Delta_{a}\right)\right|_{\Delta_{a}\left(b_{i}\right)},
$$

thus demonstrating the equivalence of $a$-maximization and volume minimization. Notice that the equivalence holds not only for the extremal value but is valid off-shell, since the two expressions in (1.2) are equal for generic values of $b_{i}$. There is an important difference between the two extremization principles. $a$-maximization is performed on the space of all $\mathrm{R}$-symmetries. This spans the three mesonic symmetries, associated with the isometries of $Y_{5}$ and a number (in principle large) of baryonic symmetries, associated with the non-trivial

\footnotetext{
${ }^{2}$ See also [6, 7] for a different approach based on five-dimensional supergravity. See also a similar approach for $c$-extremization in [8].
} 
three-cycles of $Y_{5}$. On the other hand, volume minimization is performed on the direction of the Reeb vector, spanned by $b_{i}$ and corresponding to the mesonic symmetries only. The consistency of the two extremizations is a consequence of the automatic decoupling of the baryonic symmetries from the $a$-maximization procedure in the given parameterization. This follows from the identity proved in [9]

$$
\left.\sum_{a} B_{a} \frac{\partial a\left(\Delta_{a}\right)}{\partial \Delta_{a}}\right|_{\Delta_{a}(b)} \equiv 0
$$

where $B_{a}$ is a baryonic symmetry.

After compactification on $\Sigma_{\mathfrak{g}}$ we obtain a two-dimensional theory depending on magnetic fluxes $\mathfrak{n}_{a}$ for all the symmetries of the original theory, including the baryonic ones. The exact R-symmetry can be found by extremizing the trial right-moving central charge with respect to the mesonic and baryonic symmetries $[2,3]$. There is a simple formula for the trial central charge of the $(0,2)$ CFT at large $N$, that, in the basis for R-charges of [9], reads [17]

$$
c_{r}\left(\Delta_{a}, \mathfrak{n}_{a}\right)=-\frac{32}{9} \sum_{a=1}^{d} \mathfrak{n}_{a} \frac{\partial a\left(\Delta_{a}\right)}{\partial \Delta_{a}} .
$$

In order to study the gravitational dual of $c$-extremization, the authors of $[12,13]$ defined a family of off-shell backgrounds, again depending on the Reeb vector. They also defined a functional $c\left(b_{i}, \mathfrak{n}_{a}\right)$ of the Reeb vector and fluxes whose extremization selects the on-shell R-symmetry. It has been explicitly checked in many examples in $[12,13]$ that this procedure is equivalent to $c$-extremization, and the equivalence holds off-shell. We will prove in this note that this is true in general for all toric quivers and that the proof [9] extends very nicely to the two-dimensional case. Indeed, the ingredients are exactly the same. We will define a natural parameterization of the R-charges in terms of the Reeb vector and magnetic fluxes, $\Delta_{a}\left(b_{i}, \mathfrak{n}_{a}\right)$, just by generalizing the logic behind (1.1). Then we will show that for an arbitrary toric quiver

$$
\left.c\left(b_{i}, \mathfrak{n}_{a}\right) \equiv c_{r}\left(\Delta_{a}, \mathfrak{n}_{a}\right)\right|_{\Delta_{a}(b, \mathfrak{n})} \equiv-\left.\frac{32}{9} \sum_{a=1}^{d} \mathfrak{n}_{a} \frac{\partial a\left(\Delta_{a}\right)}{\partial \Delta_{a}}\right|_{\Delta_{a}(b, \mathfrak{n})} .
$$

Moreover, as in four dimensions, the baryonic symmetries explicitly decouple from the extremization process in this parameterization

$$
\left.\sum_{a} B_{a} \frac{\partial c_{r}\left(\Delta_{a}, \mathfrak{n}_{a}\right)}{\partial \Delta_{a}}\right|_{\Delta_{a}(b, \mathfrak{n})} \equiv 0
$$

In particular, we do not see any particular difference in the role of baryonic symmetries in two dimensions compared to four.

It is also interesting to study the theories living on M2-branes at a toric Calabi-Yau four-fold $C\left(Y_{7}\right)$ and their twisted compactifications on a Riemann surface. In this case, the exact R-symmetry of the three-dimensional theory is obtained by extremizing the free energy on $S^{3}, F_{S^{3}}\left(\Delta_{a}\right)$. The equivalence of volume minimization for four-folds $[4,5]$ and the extremization of $F_{S^{3}}\left(\Delta_{a}\right)$ has been checked in many examples in [18, 19]. Given the 
complications of three dimensions and the absence of a complete classification of quiver duals to Calabi-Yau four-folds, there is no general proof. The twisted compactifications of M2-brane theories are dual in the IR to $\mathrm{AdS}_{2} \times Y_{9}$ backgrounds, where $Y_{9}$ is topologically a fibration of $Y_{7}$ over $\Sigma_{\mathfrak{g}}$. These backgrounds can be interpreted as the horizon of magnetically charged $\mathrm{AdS}_{4}$ black holes. The construction in [12] also applies to these solutions and the authors of [12] identified the quantity to extremize with the entropy of the black hole in various cases. Interestingly, it is suggested by a field theory computation [20] that the entropy of magnetically charged black holes in $\mathrm{AdS}_{4} \times Y_{7}$ should be obtained by extremizing the functional

$$
\mathcal{I}\left(\Delta_{a}, \mathfrak{n}_{a}\right)=-\frac{1}{2} \sum_{a=1}^{d} \mathfrak{n}_{a} \frac{\partial F_{S^{3}}\left(\Delta_{a}\right)}{\partial \Delta_{a}} .
$$

This is certainly true for the theory with $Y_{7}=S^{7}$ as shown in [21, 22], where a microscopic counting for the entropy of magnetically charged black holes in $\operatorname{AdS}_{4} \times S^{7}$ has been performed. We then expect that, also off-shell, the construction of [12] is dual to $\mathcal{I}$-extremization. In this note we just verify this statement for $Y_{7}=S^{7}$, reproducing the extremization of $[21,22]$ also off-shell. We leave the investigation of more general SasakiEinstein manifold $Y_{7}$, where the computation is more complicated, to the future. The microscopic computation of the entropy of black holes in $\mathrm{AdS}_{4} \times Y_{7}$ for generic $Y_{7}$ is still an open problem. In particular, baryonic symmetries enter in a puzzling way in the large $N$ limit, as noticed in $[20,23,24]$. The formalism of $[12,13]$ seems well suited to address these problems and we hope to come back to these questions in the future. Finally, notice the analogy of (1.7) with (1.4). In the context of the large $N$ limit of topologically twisted theories these identities arise as special cases of the index theorem discussed in $[17,20]$.

The note is organized as follows. In section 2 we discuss general features of fourdimensional toric quivers and their twisted compactifications. In section 3 we first review the proof of the equivalence between $a$-maximization and volume minimization for all fourdimensional toric theories and then we extend it to the equivalence between $c$-extremization and the construction in $[12,13]$. For the convenience of the reader, the technical aspects of the proof are deferred to appendix B. In section 4 we give explicit formulae for the R-charge parameterization and we present few examples. In section 5 we show that the formalism [12, 13] for $Y_{7}=S^{7}$ is equivalent off-shell to the $\mathcal{I}$-extremization principle for black holes in $\mathrm{AdS}_{4} \times S^{7}$. Finally, in appendix A we review the proof of (1.4) for the right-moving central charge $c_{r}$.

\section{Introducing the field theory}

In this section we review some general aspects of the quiver gauge theories living on D3branes at toric Calabi-Yau singularities and of their twisted compactifications on Riemann surfaces.

\section{1 $\mathcal{N}=1$ superconformal field theories}

We first discuss the four-dimensional aspect of the story. Consider the type IIB background $\mathrm{AdS}_{5} \times Y_{5}$, where $Y_{5}$ is a five-dimensional Sasaki-Einstein manifold. In the AdS/CFT 
correspondence, this is dual to the $\mathcal{N}=1$ superconformal theory living on $N$ D3-branes sitting at the tip of the Calabi-Yau cone $\mathrm{CY}_{3}=C\left(Y_{5}\right)$ with base $Y_{5}$ [25-27]. Familiar examples of Sasaki-Einstein manifolds include $T^{1,1}$, whose dual is the Klebanov-Witten theory [25], and the $\mathrm{Y}^{p, q}$ and $\mathrm{L}^{p, q, r}$ spaces [28-30], whose dual field theories have been identified in [31] and [32-34], respectively. When the $\mathrm{CY}_{3}$ is toric, there is a general prescription for constructing the gauge theory associated with the D3-branes [14-16] based on dimer models and tilings. For our purposes, we will need just some general information about the quiver, that we review following [9].

A toric affine $\mathrm{CY}_{3}$ is specified by its fan, a collections of vectors $v_{a}$ in $\mathbb{R}^{3}$ with integer entries. The Calabi-Yau condition requires that all the $v_{a}$ lie on a plane that we will take to be the plane orthogonal to the vector $e_{1}=(1,0,0)$. The toric cone is then specified by $d$ vectors $v_{a}=\left(1, \vec{v}_{a}\right)$ for $a=1, \cdots, d$. The restriction to the plane of these vectors define a regular polygon with integer vertices called the toric diagram. There is a toric divisor $D_{a}$ for each vertex. Each $D_{a}$ is a cone over a three cycle $S_{a}$ in $Y_{5}$. There are $d$ such cycles but only $d-3$ are independent in cohomology. All the data and symmetries of the gauge theory can be extracted from the geometry [14-16]. The theory has an R-symmetry and $d-1 \mathrm{U}(1)$ global symmetries that can mix with it. A particularly important role is played by the baryonic symmetries. There are precisely $d-3$ of them, corresponding to the inequivalent non-trivial three-cycles $S_{a}$ of $Y_{5}$. They are holographically dual to the $d-3$ gauge fields that we obtain by reducing the type IIB four-form potential on the three-cycles $S_{a}$. The remaining three symmetries are called mesonic and are holographically dual to the three gauge fields associated with the isometries of the toric $Y_{5}$. One is an R-symmetry and the other two are global symmetries. A convenient way to parameterize the global and R-symmetry comes from the prescription in [9] or, equivalently, from the folded quiver formalism of [32]. The $d-1$ global symmetries can be parameterized by assigning a real number $F_{a}$ to each vertex with the constraint

$$
\sum_{a=1}^{d} F_{a}=0 .
$$

In the minimal toric phase, ${ }^{3}$ the theory contains a number $|G|$ of gauge group factors $\mathrm{SU}(N)$ equal to twice the area of the toric diagram. Moreover, defining the vectors $w_{a}=v_{a+1}-v_{a}$ lying in the plane, there are precisely $\left|\left(e_{1}, w_{a}, w_{b}\right)\right|$ bi-fundamental chiral fields $\Phi_{a b}$ with charge $F_{a+1}+F_{a+2}+\ldots+F_{b}$ for each pair $(a, b)$ such that the outgoing normal of $w_{a}$ can be rotated counter-clockwise into that of $w_{b}$ in the plane with an angle smaller than $\pi{ }^{4}$ The baryonic symmetries, which we will denote by $B_{a}$, are further characterized by the vector identity

$$
\sum_{a=1}^{d} B_{a} v_{a}=0 .
$$

\footnotetext{
${ }^{3}$ There are many different quivers that describe the same IR SCFT. They are related by Seiberg dualities. The toric phases have the same number of gauge groups but different matter content. The minimal phase corresponds to the quiver with the smallest number of chiral fields.

${ }^{4}$ In this note, we will use the following notation for determinants of vectors $\left(v_{a}, v_{b}, v_{c}\right) \equiv \operatorname{det}\left(v_{a}, v_{b}, v_{c}\right)$. We also identify indices modulo $d$, so that, for example, $v_{d+1}=v_{1}$.
} 
Similarly, we can parameterize the R-charges of all fields in the quiver by assigning a number $\Delta_{a}$ to each vertex with the constraint [9]

$$
\sum_{a=1}^{d} \Delta_{a}=2
$$

The chiral fields $\Phi_{a b}$ have R-charge $\Delta_{a+1}+\Delta_{a+2}+\ldots+\Delta_{b}$.

The quiver and all interactions can be written explicitly but we will not need the explicit matter content in the following. The reader can find many examples in $[9,35]$. The only important information is that there is a very simple formula for the central charge of the CFT in the large $N$ limit. According to $a$-maximization [1], the exact central charge $a$ of the SCFT can be obtained by extremizing the trial central charge ${ }^{5}$

$$
a\left(\Delta_{a}\right)=\frac{9}{32} \operatorname{Tr} R\left(\Delta_{a}\right)^{3},
$$

where the trace runs over all the fermions of the theory and $R(\Delta)$ denotes their R-charges as a function of $\Delta_{a}$. Explicitly, we have

$$
a\left(\Delta_{a}\right)=\frac{9}{32} N^{2}\left(|G|+\sum_{\Phi_{a b}} \operatorname{mult}\left(\Phi_{a b}\right)\left(\Delta_{\Phi_{a b}}-1\right)^{3}\right),
$$

where $\operatorname{mult}\left(\Phi_{a b}\right)=\left|\left(e_{1}, v_{a}, v_{b}\right)\right|$. It has been shown in [37] that the trial central charge of the theory can be written in the large $N$ limit as

$$
a\left(\Delta_{a}\right)=\frac{9}{32} \sum_{a, b, c=1}^{d} c_{a b c} \Delta_{a} \Delta_{b} \Delta_{c}
$$

where the t'Hooft anomaly coefficients are given by

$$
c_{a b c}=\frac{N^{2}}{2}\left|\left(v_{a}, v_{b}, v_{c}\right)\right| .
$$

We can remove the absolute value if we assume an order in the toric diagram. Assuming that the vertices of the toric diagram are numerated in counter-clockwise direction, if $1 \leq a<b<c \leq d$ we can write $c_{a b c}=N^{2}\left(v_{a}, v_{b}, v_{c}\right) / 2>0$. The trial central charge can be then written as

$$
a\left(\Delta_{a}\right)=\frac{27}{32} N^{2} \sum_{1 \leq a<b<c \leq d}\left(v_{a}, v_{b}, v_{c}\right) \Delta_{a} \Delta_{b} \Delta_{c} .
$$

All our formulae are strictly valid in the large $N$ limit where $c=a$.

The extremization of (2.8) gives the exact R-charges $\bar{\Delta}_{a}$ of the fields in the SCFT. They can be compared with the predictions for the dimension of baryonic operators in the

\footnotetext{
${ }^{5} \operatorname{In}$ general $a=\frac{9}{32} \operatorname{Tr} R^{3}-\frac{3}{32} \operatorname{Tr} R^{3}$, but we work in the large $N$ limit where $c=a$ [36]. In particular, for all our quivers, in the large $N$ limit, $\operatorname{Tr} R=-16(c-a)=0$.
} 
gravity dual. The baryonic operator $\operatorname{det} \Phi_{a-1, a}$ is obtained by wrapping a D3-branes on the three-cycle $S_{a}$ and the R-charge of $\Phi_{a-1, a}$ can be computed by the standard formula [38]

$$
\bar{\Delta}_{a}=\frac{\pi \operatorname{Vol}\left(S_{a}\right)}{3 \operatorname{Vol}\left(Y_{5}\right)} .
$$

The value of the exact central charge of the CFT is also given by [39]

$$
a\left(\bar{\Delta}_{a}\right)=\frac{\pi^{3} N^{2}}{4 \operatorname{Vol}\left(Y_{5}\right)} .
$$

That $a$-maximization reproduces these formulae has been tested in many examples and it can be proved in general for all toric quivers [9].

\subsection{Twisted compactification to two dimensions}

Let us now consider the theory compactified on a Riemann surface $\Sigma_{\mathfrak{g}}$ with a topological twist and assume that it flows to a two-dimensional $\mathcal{N}=(0,2)$ CFT at low energies. The gravitational dual is a type IIB solution interpolating between $\mathrm{AdS}_{5} \times Y_{5}$ and a warped compactification $\mathrm{AdS}_{3} \times_{W} Y_{7}$ where $Y_{7}$ is topologically a fibration of $Y_{5}$ over $\Sigma_{\mathfrak{g}}[2,3]$. In general, we have a family of such two-dimensional CFTs labeled by the magnetic flux of the R-symmetry on $\Sigma_{\mathfrak{g}}$. Once again, we can parameterize the R-symmetry flux with integers $\mathfrak{n}_{a}$ associated with the vertices of the toric diagram and satisfying

$$
\sum_{a=1}^{d} \mathfrak{n}_{a}=2-2 \mathfrak{g}
$$

We will refer to this constraint as the twisting condition. It is equivalent to the requirement that the background for the R-symmetry cancels the spin connection. As shown in $[2,3]$ the right-moving central charge of the two-dimensional theory can be found by extremizing the trial right-moving central charge

$$
c_{r}\left(\Delta_{a}, \mathfrak{n}_{a}\right)=3 \operatorname{Tr} \gamma_{3} R\left(\Delta_{a}\right)^{2},
$$

where $\gamma_{3}$ is the chirality operator in two dimensions and the trace runs over all the twodimensional fermions. As shown in [17], the trial right-moving central charge of the theory compactified on $\Sigma_{\mathfrak{g}}$ in the large $N$ limit can be compactly written in terms of the fourdimensional trial $a$ charge as ${ }^{6}$

$$
\begin{aligned}
c_{r}(\Delta, \mathfrak{n}) & =-\frac{32}{9} \sum_{a=1}^{d} \mathfrak{n}_{a} \frac{\partial a(\Delta)}{\partial \Delta_{a}}=-3 \sum_{a, b, c=1}^{d} c_{a b c} \mathfrak{n}_{a} \Delta_{b} \Delta_{c} \\
& =3 N^{2} \sum_{1 \leq a<b<c \leq d}\left(v_{a}, v_{b}, v_{c}\right)\left(\mathfrak{n}_{a} \Delta_{b} \Delta_{c}+\mathfrak{n}_{b} \Delta_{a} \Delta_{c}+\mathfrak{n}_{c} \Delta_{a} \Delta_{b}\right) .
\end{aligned}
$$

This relation between $c_{r}$ and $a$ has been proven in [17] for a large class of quivers, including the toric ones, by comparing the four-dimensional and two-dimensional central charges. It

\footnotetext{
${ }^{6}$ Notice that in (2.13) we impose the constraint $\sum_{a} \Delta_{a}=2$ after differentiation. For the logic behind it see appendix A.1.
} 
has been also verified in many toric examples in [40]. It can be also obtained in a simple way by integrating the four-dimensional anomaly polynomial on $\Sigma_{\mathfrak{g}}$, following the logic in appendix $\mathrm{C}$ of [41]. We review the derivation in appendix A. The formula is valid in the large $N$ limit where $c_{r}=c_{l}=c$.

That $c$-extremization correctly reproduces the central charge predicted by the gravitational dual has been tested in many examples [2, 3, 40, 42-44].

\section{$3 \quad c$-extremization equals its gravity dual for all toric quivers}

In this section we first briefly review the equivalence of $a$-maximization with the volume minimization proposed in $[4,5]$ and then we extend it to the equivalence of $c$-extremization with the construction proposed in $[12,13]$ for all toric quiver. The technical parts of the proof are discussed in appendix B.

\section{1 $a$-maximization is volume minimization}

The gravity dual of $a$-maximization has been found in $[4,5]$ by defining a class of offshell backgrounds that solve the conditions for supersymmetry but relax the equations of motion. In particular, the authors of $[4,5]$ replace the Sasaki-Einsten metric on $Y_{5}$ with a general Sasaki metric. The metric depends on a Reeb vector which is a linear combinations of the vector fields $\partial_{\phi_{i}}$ generating the toric $\mathrm{U}(1)^{3}$ action

$$
\zeta=\sum_{i=1}^{3} b_{i} \partial_{\phi_{i}},
$$

and specifies the direction of the R-symmetry vector field inside the isometries of $Y_{5}$. Supersymmetry requires $b_{1}=3$. The volumes of Sasaki manifold $Y_{5}$ and of its three-cycles $S_{a}$ are now functions of the Reeb vector $b=\left(b_{1}, b_{2}, b_{3}\right)$

$$
\begin{aligned}
& \operatorname{Vol}\left(Y_{5}\right)=\frac{\pi^{3}}{b_{1}} \sum_{a=1}^{d} \frac{\left(v_{a-1}, v_{a}, v_{a+1}\right)}{\left(v_{a-1}, v_{a}, b\right)\left(v_{a}, v_{a+1}, b\right)}, \\
& \operatorname{Vol}\left(S_{a}\right)=2 \pi^{2} \frac{\left(v_{a-1}, v_{a}, v_{a+1}\right)}{\left(v_{a-1}, v_{a}, b\right)\left(v_{a}, v_{a+1}, b\right)} .
\end{aligned}
$$

As shown in $[4,5]$, the extremization of the function

$$
a\left(b_{i}\right)=\frac{\pi^{3} N^{2}}{4 \operatorname{Vol}\left(Y_{5}\right)},
$$

reproduces the Reeb vector $\bar{b}=\left(\bar{b}_{1}, \bar{b}_{2}, \bar{b}_{3}\right)$ and the volumes of the Sasaki-Einstein manifold. By construction, $a\left(\bar{b}_{i}\right)$ reproduces the gravitational prediction (2.10) for the exact central charge of the CFT.

The equivalence of $a$-maximization with volume minimization has been proved for all toric quivers in [9]. The proof has been simplified in [10] and generalized to other quivers 
in [11]. Following [9], we define a natural parameterization for the R-charges in terms of the Reeb vector inspired by (2.9)

$$
\Delta_{a}\left(b_{i}\right)=\frac{\pi \operatorname{Vol}\left(S_{a}\right)}{b_{1} \operatorname{Vol}\left(Y_{5}\right)}
$$

where now the volumes are the functions of $b_{i}$ given in (3.2). Notice that $\sum_{a=1}^{d} \Delta_{a}\left(b_{i}\right)=2$. One then proves that $[9,10]$

$$
\left.\left.a\left(b_{i}\right) \equiv a\left(\Delta_{a}\right)\right|_{\Delta_{a}\left(b_{i}\right)} \equiv \frac{27}{32} N^{2} \sum_{1 \leq a<b<c \leq d}\left(v_{a}, v_{b}, v_{c}\right) \Delta_{a} \Delta_{b} \Delta_{c}\right|_{\Delta_{a}\left(b_{i}\right)} .
$$

One might be puzzled by the fact that $a$-extremization is performed on $d-1$ independent parameters, while volume minimization is an extremization with respect to the Reeb vector that depends on two independent parameters only. The Reeb vector in a sense only sees the mixing of the R-symmetry with the mesonic symmetries. The point is that, as proved in [9], the trial $a$-function $a\left(\Delta_{a}\right)$ is automatically extremized

$$
\left.\sum_{a} B_{a} \frac{\partial a\left(\Delta_{a}\right)}{\partial \Delta_{a}}\right|_{\Delta_{a}(b)} \equiv 0
$$

with respect to the baryonic directions, defined by (2.2).

\section{$3.2 \quad c$-extremization is equivalent to its gravity dual}

The gravity dual of $c$-extremization has been found in [12, 13]. The solution associated with a twisted compactification of the four-dimensional CFT on $\Sigma_{\mathfrak{g}}$ is a warped background $\mathrm{AdS}_{3} \times_{W} Y_{7}$ where $Y_{7}$ is topologically a fibration of $Y_{5}$ over $\Sigma_{\mathfrak{g}}$ with a five-form flux. The authors $[12,13]$ define a family of off-shell backgrounds, depending on the Reeb vector, that solve the conditions for supersymmetry but relax the equations of motion for the five-form. They also define a functional of the Reeb vector that, upon extremization, selects the onshell R-symmetry and it becomes equal to the exact two-dimensional central charge. Here we describe the basic ingredients of the construction and we refer to $[12,13]$ for details. We will work under the assumption that the gravity background associated with $Y_{5}$ exists. This is not always the case, as discussed in $[12,13]$.

The off-shell backgrounds depend on a Reeb vector $b=\left(b_{1}, b_{2}, b_{3}\right)$, and on $d$ parameters $\lambda_{a}$ and $d$ fluxes $\mathfrak{n}_{a}$. The Reeb vector is again given by

$$
\zeta=\sum_{i=1}^{3} b_{i} \partial_{\phi_{i}}
$$

and specifies the direction of the R-symmetry vector field inside the isometries of $Y_{5}$. This time supersymmetry requires $b_{1}=2$. The parameters $\lambda_{a}$ are associated with the toric divisors $D_{a}$ and determine the Kähler class of a four-dimensional transverse slice. For simplicity, we restrict to the quasi-regular case where the quotient with respect to the 
Reeb action, $V=Y_{5} / \mathrm{U}(1)$, is a four-dimensional compact toric orbifold. Then the Kähler class of $V$ is given by

$$
\omega=-2 \pi \sum_{a=1}^{d} \lambda_{a} c_{a}
$$

where $c_{a}$ are the Poincaré dual of the restriction of $D_{a}$ to $V$. Only $d-2$ parameters $\lambda_{a}$ are independent, since there are only $d-2$ independent two-cycles in $V$ (one more than in $Y_{5}$ ). We can recover the Sasaki geometry for $\lambda_{a}=-1 / 2 b_{1} \cdot{ }^{7}$ The fluxes $\mathfrak{n}_{a}$ are also associated with the divisors $D_{a}$ and satisfy the twisting condition

$$
\sum_{a=1}^{d} \mathfrak{n}_{a}=2-2 \mathfrak{g}
$$

The $\mathfrak{n}_{a}$ are magnetic fluxes for both the three gauge fields associated with the isometries of $Y_{5}$ and the $d-3$ gauge fields coming from the reduction of the four-form potential on the $d-3$ independent three-cycles $S_{a}$. The fluxes associated with the isometries enter explicitly in the fibration of $\mathrm{SE}_{5}$ over $\Sigma_{\mathfrak{g}}$ and they can be parameterized by the integers $n^{i}=\sum_{a=1}^{d} v_{a}^{i} \mathfrak{n}_{a}$. They are associated with the mesonic symmetries of the quiver. The other $d-3$ fluxes enter in the supergravity five-form and are associated with the baryonic symmetries. The relation with the fluxes defined in [13] is $M_{a}=-\mathfrak{n}_{a} N$.

Following [13], we define the master volume of the five-manifold with Kähler class (3.8)

$$
\mathcal{V}=4 \pi^{3} \sum_{a=1}^{d} \lambda_{a} \frac{\lambda_{a-1}\left(v_{a}, v_{a+1}, b\right)-\lambda_{a}\left(v_{a-1}, v_{a+1}, b\right)+\lambda_{a+1}\left(v_{a-1}, v_{a}, b\right)}{\left(v_{a-1}, v_{a}, b\right)\left(v_{a}, v_{a+1}, b\right)}
$$

Notice that we identify indices modulo $d$ so that $v_{d+1}=v_{1}$ and $\lambda_{d+1}=\lambda_{1}$. The supersymmetry and flux quantization conditions for the off-shell background can be then summarized by $[13]^{8}$

$$
\begin{aligned}
N & =-\sum_{a=1}^{d} \frac{\partial \mathcal{V}}{\partial \lambda_{a}} \\
\mathfrak{n}_{a} N & =-\frac{A}{2 \pi} \sum_{b=1}^{d} \frac{\partial^{2} \mathcal{V}}{\partial \lambda_{a} \partial \lambda_{b}}-b_{1} \sum_{i=1}^{3} n^{i} \frac{\partial^{2} \mathcal{V}}{\partial \lambda_{a} \partial b_{i}}, \\
A \sum_{a, b=1}^{d} \frac{\partial^{2} \mathcal{V}}{\partial \lambda_{a} \partial \lambda_{b}} & =2 \pi n^{1} \sum_{a=1}^{d} \frac{\partial \mathcal{V}}{\partial \lambda_{a}}-2 \pi b_{1} \sum_{i=1}^{3} n^{i} \sum_{a=1}^{d} \frac{\partial^{2} \mathcal{V}}{\partial \lambda_{a} \partial b_{i}}
\end{aligned}
$$

where $n^{i}=\sum_{a=1}^{d} v_{a}^{i} \mathfrak{n}_{a}$. As shown in [13] and reviewed in section B.1, $\mathcal{V}$ is a function of only $d-2$ independent parameters $\lambda_{a}$ and only $d-1$ equations in (3.11) are independent.

\footnotetext{
${ }^{7}$ In the Sasaki case, the contact form $\eta$ associated with the Reeb vector $b$ satisfies $\mathrm{d} \eta=2 \omega$ and $b_{1}=3[4,5]$. Here instead $[\mathrm{d} \eta]=[\rho] / b_{1}$ in cohomology, where the Ricci form is given by $\rho=2 \pi \sum_{a=1}^{d} c_{a}$ and $b_{1}=2$.

${ }^{8}$ In the notation of [13], we set $L^{4}=2\left(2 \pi l_{s}\right)^{4} g_{s}$. In order to compare with [13] one must also set $\Delta_{a}=\frac{R_{a}}{N}$ and $\mathfrak{n}_{a}=-\frac{M_{a}}{N}$.
} 
We can use the constraints (3.11) to eliminate the $d-2$ independent $\lambda_{a}$ and $A$ and write them as functions of $b_{i}$ and $\mathfrak{n}_{a} \cdot{ }^{9}$ We then obtain the $c$-functional [13]

$$
c\left(b_{i}, \mathfrak{n}_{a}\right)=-\left.48 \pi^{2}\left(A \sum_{a=1}^{d} \frac{\partial \mathcal{V}}{\partial \lambda_{a}}+2 \pi b_{1} \sum_{i=1}^{3} n^{i} \frac{\partial \mathcal{V}}{\partial b_{i}}\right)\right|_{\lambda_{a}(b, \mathfrak{n}), A(b, \mathfrak{n})} .
$$

For further reference, we also define the on-shell value of the master volume

$$
\mathcal{V}_{\text {on-shell }}\left(b_{i}, \mathfrak{n}_{a}\right)=\left.\mathcal{V}\right|_{\lambda_{a}(b, \mathfrak{n}), A(b, \mathfrak{n})} .
$$

The authors of [13] checked that the extremization of $c$ with respect to $b_{i}$ (with $b_{1}=2$ ) correctly reproduces the central charge of the two-dimensional CFT in various examples, including the $\mathrm{Y}^{p, q}$ and $\mathrm{X}^{p, q}$ manifolds. By an explicit computation along the lines of $[2$, $3,42]$, they also show that the identification holds off-shell and $c\left(b_{i}, \mathfrak{n}_{a}\right)$ can be identified with the trial right-moving central charge. We want now to show that this holds for all toric quiver, using the expression (2.13) derived in [17] and a natural parameterization of the R-charges based on the toric data.

In order to prove this, in analogy with [9] and the four-dimensional case, we define

$$
\Delta_{a}\left(b_{i}, \mathfrak{n}_{a}\right)=-\left.\frac{2}{N} \frac{\partial \mathcal{V}}{\partial \lambda_{a}}\right|_{\lambda_{a}(b, \mathfrak{n}), A(b, \mathfrak{n})}
$$

satisfying $\sum_{a} \Delta_{a}=2$. This expression is indeed the holographic prediction for the Rcharges of baryonic operators obtained by wrapping D3-branes on the cycles associated with the toric divisors $D_{a}[13,45]$. As such this is the natural generalization of the fourdimensional parameterization (3.4). It is important to observe that the $\Delta_{a}$ satisfy [13]

$$
\sum_{a=1}^{d} \Delta_{a} v_{a}=\left.2 \frac{b}{b_{1}}\right|_{\Delta_{a}(b, \mathfrak{n})}
$$

We will show how to obtain an explicit expression for $\Delta_{a}\left(b_{i}, \mathfrak{n}_{a}\right)$ in section 4 .

With these definitions, we will show that ${ }^{10}$

$$
\left.c\left(b_{i}, \mathfrak{n}_{a}\right) \equiv c_{r}\left(\Delta_{a}, \mathfrak{n}_{a}\right)\right|_{\Delta_{a}(b, \mathfrak{n})} \equiv-\left.\frac{32}{9} \sum_{a=1}^{d} \mathfrak{n}_{a} \frac{\partial a\left(\Delta_{a}\right)}{\partial \Delta_{a}}\right|_{\Delta_{a}(b, \mathfrak{n})},
$$

thus proving the off-shell equivalence of $c$-extremization and the formalism of [13] for all toric quivers.

As in four dimensions, one might be puzzled by the fact that $c$-extremization is performed on $d-1$ independent parameters, while the construction in [13] is an extremization with respect to the Reeb vector that depends on only two independent parameters. The point is again that the trial $c$-function $c_{r}\left(\Delta_{a}, \mathfrak{n}_{a}\right)$ is automatically extremized

$$
\left.\sum_{a} B_{a} \frac{\partial c_{r}(\Delta, \mathfrak{n})}{\partial \Delta_{a}}\right|_{\Delta_{a}(b, \mathfrak{n})}=\left.\sum_{a, b} B_{a} \mathfrak{n}_{b} \frac{\partial^{2} a\left(\Delta_{a}\right)}{\partial \Delta_{a} \partial \Delta_{b}}\right|_{\Delta_{a}(b, \mathfrak{n})} \equiv 0
$$

\footnotetext{
${ }^{9}$ The dependence on the remaining two variables $\lambda_{a}$ drops out from every physical quantity. To simplify the computation, one can also choose a gauge like in appendix B.2.

${ }^{10}$ In this and the following equations we set $b_{1}=2$. Reinstating $b_{1}$ we have $\mathcal{V}=\frac{b_{1}}{108 \pi^{3}} a$ and $c=\frac{b_{1}}{2} c_{r}$.
} 
with respect to the baryonic directions, defined by (2.2), as we will show. Again, this is completely analogous to [9].

Indeed, (3.16) and (3.17) follow at once from the result in appendix B, where we will prove that there exists a vector $t$ such that

$$
-6 \sum_{b, c=1}^{d} c_{a b c} \mathfrak{n}_{b} \Delta_{c}=c_{r}(\Delta, \mathfrak{n})+\left.\left(e_{1}, r_{a}, t\right)\right|_{\Delta_{a}(b, \mathfrak{n})},
$$

where $r_{a}=v_{a}-b / b_{1}$. (3.16) follows by multiplying (3.18) by $\Delta_{a}$ and summing over $a$. The term with the vector $t$ cancels since $\sum_{a} \Delta_{a} r_{a}=0$, as a consequence of (3.15). Similarly, (3.17) follows by multiplying (3.18) by $B_{a}$ and summing over $a$. The term with $c_{r}$ on the right hand side vanishes because $\sum_{a} B_{a}=0$ and the term with $t$ because $\sum_{a} B_{a} r_{a}=0$, where we used (2.2).

Let us also observe that, quite interestingly, the on-shell value of the master volume coincides with the four-dimensional trial central charge

$$
\left.\mathcal{V}_{\text {on-shell }}\left(b_{i}, \mathfrak{n}_{a}\right) \equiv \frac{1}{54 \pi^{3}} a\left(\Delta_{a}\right)\right|_{\Delta_{a}(b, \mathfrak{n})} .
$$

A word of caution is in order. To find the exact right-moving central charge of the two-dimensional CFT we need to extremize $c\left(b_{i}, \mathfrak{n}_{a}\right)$ with respect to $b_{2}$ and $b_{3}$ after setting $b_{1}=2$, or equivalently, $c\left(\Delta_{a}, \mathfrak{n}_{a}\right)$ with respect to $\Delta_{a}$ with the constraint $\sum_{a} \Delta_{a}=2$. Our results guarantee that the two procedures are equivalent for all toric quivers. However they do not guarantee that the exact central charge found in this way really corresponds to an IR CFT. Similarly, in gravity, nothing guarantee that the family of backgrounds discussed in $[12,13]$ contains an actual solution of the equations of motion of type IIB. Explicit examples of possible obstructions are discussed in [12].

\section{Formulae for the R-charges and examples}

In this section we discuss how to solve equations (3.11). Fortunately, there is no need of solving explicitly (3.11) in order to write the R-charges $\Delta_{a}\left(b_{i}, \mathfrak{n}_{a}\right)$. Indeed there is an explicit expression for $\Delta_{a}\left(b_{i}, \mathfrak{n}_{a}\right)$ in terms of the toric data and the fluxes $\mathfrak{n}_{a}$. Moreover, in a convenient gauge, we can also write a general expression for the solutions $\lambda_{a}$ and $A$ that allows to write $c\left(b_{i}, \mathfrak{n}_{a}\right)$ and $\mathcal{V}\left(b_{i}, \mathfrak{n}_{a}\right)$. We summarize here the result referring to appendix B for the proof. We also discuss some explicit examples.

We first show how to find the R-charges $\Delta_{a}\left(b_{i}, \mathfrak{n}_{a}\right)$ in terms of the toric data and the fluxes $\mathfrak{n}_{a}$. A consequence of (3.11) is the set of equations

$$
\begin{aligned}
& \left(v_{a-1}, v_{a}, v_{a+1}\right)\left(v_{a+1}, v_{a+2}, n\right) \Delta_{a+1}\left(b_{i}, \mathfrak{n}_{a}\right)-\left(v_{a}, v_{a+1}, v_{a+2}\right)\left(v_{a-1}, v_{a}, n\right) \Delta_{a}\left(b_{i}, \mathfrak{n}_{a}\right) \\
& =-\frac{2}{b_{1}}\left(\left(v_{a-1}, v_{a}, v_{a+1}\right)\left(v_{a+1}, v_{a+2}, b\right) \mathfrak{n}_{a+1}-\left(v_{a}, v_{a+1}, v_{a+2}\right)\left(v_{a-1}, v_{a}, b\right) \mathfrak{n}_{a}\right)
\end{aligned}
$$

where, as usual, we identify the indices modulo $d$. These equations allow to find explicitly $\Delta_{a}\left(b_{i}, \mathfrak{n}_{a}\right)$ by recursion. We can use them to express $\Delta_{a}$ in terms of $\Delta_{1}$, and, finally, determine $\Delta_{1}$ using the constraint $\sum_{a=1}^{d} \Delta_{a}=2$. 
In order to write $c\left(b_{i}, \mathfrak{n}_{a}\right)$ and $\mathcal{V}\left(b_{i}, \mathfrak{n}_{a}\right)$ we also need to solve (3.11) for the variables $\lambda_{a}$ and $A$. As already mentioned, only $d-2$ variables $\lambda_{a}$ are independent. Indeed, the master volume (3.10) is a quadratic form in $\lambda_{a}$ invariant under

$$
\lambda_{a} \rightarrow \lambda_{a}+\sum_{i=2}^{3} l_{i}\left(b_{1} v_{a}^{i}-b_{i}\right),
$$

for arbitrary functions $l_{2}$ and $l_{2}$. We can use this freedom to choose a gauge, for example $\lambda_{1}=\lambda_{2}=0$. In this gauge, we can explicitly invert the relation (3.14) and write

$$
\lambda_{a}=-\frac{N}{16 \pi^{3}} \sum_{c=2}^{a}\left(v_{c}, v_{a}, b\right) \Delta_{c}\left(b_{i}, \mathfrak{n}_{a}\right), \quad a=3, \ldots, d
$$

In this gauge, equations (3.11) also imply

$$
A=-\frac{N}{8 \pi^{2}} \frac{\left(v_{2}, v_{3}, n\right)\left(v_{1}, v_{2}, b\right)}{\left(v_{1}, v_{2}, v_{3}\right)} \Delta_{2}\left(b_{i}, \mathfrak{n}_{a}\right)-\frac{N}{4 \pi^{2} b_{1}} \frac{\left(v_{2}, v_{3}, b\right)\left(v_{1}, v_{2}, b\right)}{\left(v_{1}, v_{2}, v_{3}\right)} \mathfrak{n}_{2} .
$$

Finally, the $c$-functional can be simplified to

$$
c\left(b_{i}, \mathfrak{n}_{a}\right)=48 \pi^{2} N\left(\frac{A}{2}+\pi \sum_{a=1}^{d} \lambda_{a} \mathfrak{n}_{a}\right) .
$$

As clear from the previous formulae and manifest in the following examples, the gauge invariant quantities $\Delta_{a}\left(b_{i}, \mathfrak{n}_{a}\right), \mathcal{V}\left(b_{i}, \mathfrak{n}_{a}\right)$ and $c\left(b_{i}, \mathfrak{n}_{a}\right)$ are homogeneous polynomials of $b_{i} / b_{1}$. In particular, $\Delta_{a}$ is a linear homogeneous polynomial in $b_{i} / b_{1}, c_{r} / b_{1}$ is quadratic and $\mathcal{V} / b_{1}$ is cubic. In the gauge where two $\lambda_{a}$ are set to zero, also $\lambda_{a} / b_{1}$ and $A / b_{1}$ are quadratic homogeneous polynomials of $b_{i} / b_{1}$. Setting $b_{1}=2$, as required by supersymmetry, $\Delta_{a}$ becomes a linear function of $b_{2}$ and $b_{3}$ with rational functions of the fluxes as coefficients. This should be contrasted with the case of $a$-maximization [9], where the R-charges $\Delta_{a}\left(b_{i}\right)$ are rational functions of $b$ with poles on the sides of the toric diagram, as one can see from (3.4) and (3.2).

We now present few examples.

\section{$4.1 \mathcal{N}=4 \mathrm{SYM}$}

Our first example is the $\mathcal{N}=4$ super Yang-Mills (SYM) theory compactified on $\Sigma_{\mathfrak{g}}$. The holographic dual has been found in [3]. The manifold is $Y_{5}=S^{5}$ and the toric cone is specified by the vectors

$$
\vec{v}_{1}=(0,0), \quad \vec{v}_{2}=(1,0), \quad \vec{v}_{3}=(0,1) .
$$

In $\mathcal{N}=1$ notation, the four-dimensional theory contains three adjoint chiral fields $\Phi_{a}$, $a=1,2,3$, with superpotential

$$
W=\operatorname{Tr}\left(\Phi_{3}\left[\Phi_{1}, \Phi_{2}\right]\right)
$$


In this example, the vertices are in one-to-one correspondence with the fields and fluxes. The vertex $v_{a}$ is associated with the field $\Phi_{a}$ with R-charge $\Delta_{a}$ and the flux $\mathfrak{n}_{a}$. They satisfy

$$
\sum_{a=1}^{3} \Delta_{a}=2, \quad \sum_{a=1}^{3} \mathfrak{n}_{a}=2-2 \mathfrak{g}
$$

which just express the fact that the superpotential (4.7) has R-charge two. Since $d=3$, there are no baryonic symmetries. The trial central charge $a$, at large $N$, reads (cf. (2.5) and $(2.8))$

$$
a\left(\Delta_{a}\right)=\frac{9}{32} N^{2}\left(1+\sum_{a=1}^{3}\left(\Delta_{a}-1\right)^{3}\right)=\frac{27}{32} N^{2} \Delta_{1} \Delta_{2} \Delta_{3} .
$$

The trial central charge $c_{r}$ is given by (2.13)

$$
c_{r}\left(\Delta_{a}, \mathfrak{n}_{a}\right)=-3 N^{2}\left(\Delta_{1} \Delta_{2} \mathfrak{n}_{3}+\Delta_{2} \Delta_{3} \mathfrak{n}_{1}+\Delta_{1} \Delta_{3} \mathfrak{n}_{2}\right) .
$$

Solving explicitly (3.11) or using the recursion relations (4.1) we find

$$
\Delta_{1}\left(b_{i}\right)=\frac{2}{b_{1}}\left(b_{1}-b_{2}-b_{3}\right), \quad \Delta_{2}\left(b_{i}\right)=\frac{2 b_{2}}{b_{1}}, \quad \Delta_{3}\left(b_{i}\right)=\frac{2 b_{3}}{b_{1}} .
$$

Notice that $\Delta_{a}\left(b_{i}\right)$ are independent of the fluxes $\mathfrak{n}_{a}$. This is due to the absence of baryonic symmetries. Moreover, comparing with (3.2) we see that

$$
\Delta_{a}\left(b_{i}\right) \equiv \frac{\pi}{b_{1}} \frac{\operatorname{Vol}\left(S_{a}\right)}{\operatorname{Vol}\left(Y_{5}\right)} .
$$

Therefore, for $\mathcal{N}=4 \mathrm{SYM}$ the parameterization (3.14) coincides with the one used in [9] for $a$-maximization. Moreover, the on-shell value of the master volume is given by

$$
\mathcal{V}_{\text {on-shell }}\left(b_{i}\right)=\frac{N^{2}}{16 b_{1}^{2} \operatorname{Vol}\left(S^{5}\right)},
$$

and again is independent of $\mathfrak{n}_{a}$. Setting $b_{1}=2$ we find the very simple identification

$$
\Delta_{1}=2-b_{2}-b_{3}, \quad \Delta_{2}=b_{2}, \quad \Delta_{3}=b_{3} .
$$

One can easily verify that (3.16) and (3.19) are satisfied. For $\mathcal{N}=4$ SYM, (3.19) is just equivalent to the equivalence of $a$-maximization and volume minimization found in [9], since (4.13) holds and the parameterization of R-charges is the same in two and four dimensions.

\subsection{Klebanov-Witten theory}

Our second example is the twisted compactification of the Klebanov-Witten theory [25] on $\Sigma_{\mathfrak{g}}$, discussed e.g. in $[17,42]$. The manifold in this case is $Y_{5}=T^{1,1}$. The toric cone $C\left(T^{1,1}\right)$ is determined by the vectors

$$
\vec{v}_{1}=(0,0), \quad \vec{v}_{2}=(1,0), \quad \vec{v}_{3}=(1,1), \quad \vec{v}_{4}=(0,1) .
$$


This theory has $\mathcal{N}=1$ supersymmetry. The quiver contains two $\mathrm{SU}(N)$ gauge groups with two bi-fundamental chiral fields $A_{i}$ in the representation $(\mathbf{N}, \overline{\mathbf{N}})$ and two bi-fundamental chiral fields $B_{i}$ in the representation $(\overline{\mathbf{N}}, \mathbf{N})$. The theory has a quartic superpotential

$$
W=\operatorname{Tr}\left(A_{1} B_{1} A_{2} B_{2}-A_{1} B_{2} A_{2} B_{1}\right) .
$$

We introduce four chemical potentials $\Delta_{a}$ and fluxes $\mathfrak{n}_{a}$, one for each of the four fields $\left\{A_{i}, B_{i}\right\}$, associated with the four vertices $v_{a}$, with the constraints

$$
\sum_{a=1}^{4} \Delta_{a}=2, \quad \sum_{a=1}^{4} \mathfrak{n}_{a}=2-2 \mathfrak{g} .
$$

The trial $a$ central charge can be computed from either (2.5) or (2.8) and it reads

$$
a\left(\Delta_{a}\right)=\frac{27}{32} N^{2}\left(\Delta_{1} \Delta_{2} \Delta_{3}+\Delta_{1} \Delta_{2} \Delta_{4}+\Delta_{1} \Delta_{3} \Delta_{4}+\Delta_{2} \Delta_{3} \Delta_{4}\right) .
$$

The trial central charge $c_{r}$ is given by $(2.13)$

$$
c_{r}\left(\Delta_{a}, \mathfrak{n}_{a}\right)=-3 N^{2} \sum_{\substack{a<b \\(a, b) \neq c}}^{4} \Delta_{a} \Delta_{b} \mathfrak{n}_{c}
$$

Solving explicitly (3.11) or using the recursion relations (4.1) we find

$$
\begin{aligned}
& \Delta_{1}\left(b_{i}, \mathfrak{n}_{a}\right)=\frac{2}{b_{1}} \frac{b_{1}\left(\mathfrak{n}_{1}+\mathfrak{n}_{2}+\mathfrak{n}_{4}\right)-b_{2}\left(\mathfrak{n}_{1}+\mathfrak{n}_{2}\right)-b_{3}\left(\mathfrak{n}_{1}+\mathfrak{n}_{4}\right)}{\mathfrak{n}_{1}+\mathfrak{n}_{2}+\mathfrak{n}_{3}+\mathfrak{n}_{4}}, \\
& \Delta_{2}\left(b_{i}, \mathfrak{n}_{a}\right)=\frac{2}{b_{1}} \frac{b_{2}\left(\mathfrak{n}_{1}+\mathfrak{n}_{2}\right)+b_{1} \mathfrak{n}_{3}-b_{3}\left(\mathfrak{n}_{2}+\mathfrak{n}_{3}\right)}{\mathfrak{n}_{1}+\mathfrak{n}_{2}+\mathfrak{n}_{3}+\mathfrak{n}_{4}} \\
& \Delta_{3}\left(b_{i}, \mathfrak{n}_{a}\right)=\frac{2}{b_{1}} \frac{-b_{1} \mathfrak{n}_{3}+b_{3}\left(\mathfrak{n}_{2}+\mathfrak{n}_{3}\right)+b_{2}\left(\mathfrak{n}_{3}+\mathfrak{n}_{4}\right)}{\mathfrak{n}_{1}+\mathfrak{n}_{2}+\mathfrak{n}_{3}+\mathfrak{n}_{4}} \\
& \Delta_{4}\left(b_{i}, \mathfrak{n}_{a}\right)=\frac{2}{b_{1}} \frac{b_{1} \mathfrak{n}_{3}+b_{3}\left(\mathfrak{n}_{1}+\mathfrak{n}_{4}\right)-b_{2}\left(\mathfrak{n}_{3}+\mathfrak{n}_{4}\right)}{\mathfrak{n}_{1}+\mathfrak{n}_{2}+\mathfrak{n}_{3}+\mathfrak{n}_{4}}
\end{aligned}
$$

Notice that these are linear polynomials in $b_{i} / b_{1}$.

The baryonic symmetry $\mathrm{U}(1)_{B}$ is characterized by $(2.2)$ and it is given by

$$
B_{1}=-1, \quad B_{2}=1, \quad B_{3}=-1, \quad B_{4}=1 .
$$

Thus, the decoupling condition (3.17) can be explicitly written as

$$
\Delta_{1} \mathfrak{n}_{3}+\Delta_{3} \mathfrak{n}_{1}-\Delta_{2} \mathfrak{n}_{4}-\Delta_{4} \mathfrak{n}_{2}=0
$$

One can check that (4.22) is automatically satisfied by the solution (4.20). 
Finally, $c\left(b_{i}, \mathfrak{n}_{a}\right)$ and $\mathcal{V}_{\text {on-shell }}\left(b_{i}, \mathfrak{n}_{a}\right)$ read

$$
\begin{aligned}
\mathcal{V}_{\text {on-shell }}\left(b_{i}, \mathfrak{n}_{a}\right)= & \frac{N^{2}}{16 \pi^{3} b_{1}^{2}\left(\mathfrak{n}_{1}+\mathfrak{n}_{2}+\mathfrak{n}_{3}+\mathfrak{n}_{4}\right)^{2}}\left[2 b_{1}^{2} \mathfrak{n}_{3}\left(b_{3}\left(\mathfrak{n}_{2}+\mathfrak{n}_{3}\right)+b_{2}\left(\mathfrak{n}_{3}+\mathfrak{n}_{4}\right)\right)\right. \\
& -b_{1}\left(b_{3}^{2}\left(\mathfrak{n}_{2}+\mathfrak{n}_{3}\right)^{2}+b_{2}^{2}\left(\mathfrak{n}_{3}+\mathfrak{n}_{4}\right)^{2}+b_{1}^{2} \mathfrak{n}_{3}^{2}\right) \\
& +b_{1} b_{2} b_{3}\left(\mathfrak{n}_{1}^{2}+2\left(\mathfrak{n}_{2}+\mathfrak{n}_{4}\right) \mathfrak{n}_{1}+\mathfrak{n}_{2}^{2}-3 \mathfrak{n}_{3}^{2}+\mathfrak{n}_{4}^{2}-2 \mathfrak{n}_{2} \mathfrak{n}_{3}-2 \mathfrak{n}_{3} \mathfrak{n}_{4}\right) \\
& \left.-b_{2} b_{3}\left(\mathfrak{n}_{1}+\mathfrak{n}_{2}+\mathfrak{n}_{3}+\mathfrak{n}_{4}\right)\left(b_{2}\left(\mathfrak{n}_{1}+\mathfrak{n}_{2}-\mathfrak{n}_{3}-\mathfrak{n}_{4}\right)+b_{3}\left(\mathfrak{n}_{1}-\mathfrak{n}_{2}-\mathfrak{n}_{3}+\mathfrak{n}_{4}\right)\right)\right] \\
c\left(b_{i}, \mathfrak{n}_{a}\right)= & \frac{6 N^{2}}{b_{1}\left(\mathfrak{n}_{1}+\mathfrak{n}_{2}+\mathfrak{n}_{3}+\mathfrak{n}_{4}\right)}\left[b_{2}^{2}\left(\mathfrak{n}_{1}+\mathfrak{n}_{2}\right)\left(\mathfrak{n}_{3}+\mathfrak{n}_{4}\right)\right. \\
& -b_{2}\left(b_{1}\left(\mathfrak{n}_{1}+\mathfrak{n}_{2}-\mathfrak{n}_{3}+\mathfrak{n}_{4}\right)\left(\mathfrak{n}_{3}+\mathfrak{n}_{4}\right)+b_{3}\left(\mathfrak{n}_{1}^{2}-\mathfrak{n}_{2}^{2}+\mathfrak{n}_{3}^{2}-\mathfrak{n}_{4}^{2}\right)\right) \\
& \left.-b_{1}^{2} \mathfrak{n}_{3}^{2}-b_{3}^{2}\left(\mathfrak{n}_{2}+\mathfrak{n}_{3}\right)\left(\mathfrak{n}_{1}+\mathfrak{n}_{4}\right)+b_{1} b_{3}\left(\mathfrak{n}_{2}+\mathfrak{n}_{3}\right)\left(\mathfrak{n}_{1}+\mathfrak{n}_{2}-\mathfrak{n}_{3}+\mathfrak{n}_{4}\right)\right]
\end{aligned}
$$

One can explicitly verify that (3.16) and (3.19) are satisfied. Recall that the central charge is obtained by extremizing $c\left(b_{i}, \mathfrak{n}_{a}\right)$ with respect to $b_{2}$ and $b_{3}$ after setting $b_{2}=2$.

\section{3 $\quad Y^{p, q}$ quiver gauge theory}

Our third example is the $Y^{p, q}(p>0$ and $p \geq q \geq 0)$ quiver gauge theory [31]. The dual of the twisted compactification on $\Sigma_{\mathfrak{g}}$ is discussed in [42]. The cone $C\left(Y^{p, q}\right)$ determines a polytope with four vertices [46]

$$
\vec{v}_{1}=(0,0), \quad \vec{v}_{2}=(1,0), \quad \vec{v}_{3}=(0, p), \quad \vec{v}_{4}=(-1, p+q) .
$$

The $Y^{p, q}$ quiver has $2 p \mathrm{SU}(N)$ gauge groups with $4 p+2 q$ chiral fields $\left\{Y, Z, U^{\alpha}, V^{\alpha}\right\}$, $\alpha=1,2$, in bi-fundamental representations of pairs of gauge groups. In (4.25) we present the R-charges and their multiplicity.

\begin{tabular}{c|c|c|c}
$(a, b)$ in $\Phi_{a b}$ & multiplicity & $\mathrm{U}(1)_{R}$ & fields \\
\hline$(4,1)$ & $p+q$ & $\Delta_{1}$ & $Y$ \\
$(1,2)$ & $p$ & $\Delta_{2}$ & $U^{1}$ \\
$(2,3)$ & $p-q$ & $\Delta_{3}$ & $Z$ \\
$(3,4)$ & $p$ & $\Delta_{4}$ & $U^{2}$ \\
$(1,3)$ & $q$ & $\Delta_{2}+\Delta_{3}$ & $V^{1}$ \\
$(2,4)$ & $q$ & $\Delta_{3}+\Delta_{4}$ & $V^{2}$
\end{tabular}

Supersymmetry imposes the constraints

$$
\sum_{a=1}^{4} \Delta_{a}=2, \quad \sum_{a=1}^{4} \mathfrak{n}_{a}=2-2 \mathfrak{g} .
$$

The $a$ central charge can be computed from either (2.5) or (2.8) and it is given by

$$
\left.\frac{32}{27 N^{2}} a\left(\Delta_{a}\right)=\Delta_{2}\left(\Delta_{1}-\Delta_{3}\right) \Delta_{4} q+\left[\Delta_{2} \Delta_{3} \Delta_{4}+\Delta_{1} \Delta_{3} \Delta_{4}+\Delta_{1} \Delta_{2} \Delta_{3}+\Delta_{1} \Delta_{2} \Delta_{4}\right)\right] p
$$


Solving explicitly (3.11) or using the recursion relations (4.1) we find

$$
\begin{aligned}
& \Delta_{1}\left(b_{i}, \mathfrak{n}_{a}\right)=\frac{2}{b_{1}} \frac{p^{3}\left(b_{1}\left(\mathfrak{n}_{1}+\mathfrak{n}_{2}+\mathfrak{n}_{4}\right)-b_{2}\left(\mathfrak{n}_{1}+\mathfrak{n}_{2}\right)\right)+p q^{2}\left(b_{2} \mathfrak{n}_{2}-b_{1}\left(\mathfrak{n}_{2}+\mathfrak{n}_{4}\right)\right)}{p\left(\left(\mathfrak{n}_{1}+\mathfrak{n}_{2}+\mathfrak{n}_{3}+\mathfrak{n}_{4}\right) p^{2}+\left(\mathfrak{n}_{1}-\mathfrak{n}_{3}\right) p q-\left(\mathfrak{n}_{2}+\mathfrak{n}_{4}\right) q^{2}\right)} \\
& -\frac{2}{b_{1}} \frac{p^{2}\left(b_{3}\left(2 \mathfrak{n}_{1}+\mathfrak{n}_{2}+\mathfrak{n}_{4}\right)-b_{1} \mathfrak{n}_{1} q+b_{2}\left(\mathfrak{n}_{1}+\mathfrak{n}_{4}\right) q\right)-q^{2}\left(b_{3}\left(\mathfrak{n}_{2}+\mathfrak{n}_{4}\right)+b_{2} \mathfrak{n}_{4} q\right)}{p\left(\left(\mathfrak{n}_{1}+\mathfrak{n}_{2}+\mathfrak{n}_{3}+\mathfrak{n}_{4}\right) p^{2}+\left(\mathfrak{n}_{1}-\mathfrak{n}_{3}\right) p q-\left(\mathfrak{n}_{2}+\mathfrak{n}_{4}\right) q^{2}\right)}, \\
& \Delta_{2}\left(b_{i}, \mathfrak{n}_{a}\right)=\frac{2}{b_{1}} \frac{p^{2}\left(b_{1} \mathfrak{n}_{3}+b_{2}\left(\mathfrak{n}_{1}+\mathfrak{n}_{2}\right)\right)+\left(\mathfrak{n}_{1}-\mathfrak{n}_{3}\right) p\left(b_{2} q+b_{3}\right)-b_{2} \mathfrak{n}_{2} q^{2}}{\left(\mathfrak{n}_{1}+\mathfrak{n}_{2}+\mathfrak{n}_{3}+\mathfrak{n}_{4}\right) p^{2}+\left(\mathfrak{n}_{1}-\mathfrak{n}_{3}\right) p q-\left(\mathfrak{n}_{2}+\mathfrak{n}_{4}\right) q^{2}}, \\
& \Delta_{3}\left(b_{i}, \mathfrak{n}_{a}\right)=\frac{2}{b_{1}} \frac{p^{3}\left(b_{2}\left(\mathfrak{n}_{3}+\mathfrak{n}_{4}\right)-b_{1} \mathfrak{n}_{3}\right)+p^{2}\left(b_{3}\left(\mathfrak{n}_{2}+2 \mathfrak{n}_{3}+\mathfrak{n}_{4}\right)-b_{1} \mathfrak{n}_{3} q+b_{2}\left(\mathfrak{n}_{3}+\mathfrak{n}_{4}\right) q\right)}{p\left(\left(\mathfrak{n}_{1}+\mathfrak{n}_{2}+\mathfrak{n}_{3}+\mathfrak{n}_{4}\right) p^{2}+\left(\mathfrak{n}_{1}-\mathfrak{n}_{3}\right) p q-\left(\mathfrak{n}_{2}+\mathfrak{n}_{4}\right) q^{2}\right)} \\
& -\frac{2}{b_{1}} \frac{b_{2} \mathfrak{n}_{4} p q^{2}+q^{2}\left(b_{3}\left(\mathfrak{n}_{2}+\mathfrak{n}_{4}\right)+b_{2} \mathfrak{n}_{4} q\right)}{p\left(\left(\mathfrak{n}_{1}+\mathfrak{n}_{2}+\mathfrak{n}_{3}+\mathfrak{n}_{4}\right) p^{2}+\left(\mathfrak{n}_{1}-\mathfrak{n}_{3}\right) p q-\left(\mathfrak{n}_{2}+\mathfrak{n}_{4}\right) q^{2}\right)}, \\
& \Delta_{4}\left(b_{i}, \mathfrak{n}_{a}\right)=\frac{2}{b_{1}} \frac{p^{2}\left(b_{1} \mathfrak{n}_{3}-b_{2}\left(\mathfrak{n}_{3}+\mathfrak{n}_{4}\right)\right)+b_{3}\left(\mathfrak{n}_{1}-\mathfrak{n}_{3}\right) p+b_{2} \mathfrak{n}_{4} q^{2}}{\left(\mathfrak{n}_{1}+\mathfrak{n}_{2}+\mathfrak{n}_{3}+\mathfrak{n}_{4}\right) p^{2}+\left(\mathfrak{n}_{1}-\mathfrak{n}_{3}\right) p q-\left(\mathfrak{n}_{2}+\mathfrak{n}_{4}\right) q^{2}} .
\end{aligned}
$$

Notice that these are linear polynomials in $b_{i} / b_{1}$. At the end of the computation we can set $b_{1}=2$ as required by supersymmetry.

The baryonic symmetry $\mathrm{U}(1)_{B}$ is characterized by (2.2). It reads

$$
B_{1}=-\frac{p-q}{p}, \quad B_{2}=1, \quad B_{3}=-\frac{p+q}{p}, \quad B_{4}=1 .
$$

Hence, the decoupling condition (3.17) can be explicitly written as

$$
\Delta_{1} \mathfrak{n}_{2} p+\Delta_{3} \mathfrak{n}_{1} p+\mathfrak{n}_{2} \Delta_{4}\left(\frac{q^{2}}{p}-p\right)+\mathfrak{n}_{4} \Delta_{2}\left(\frac{q^{2}}{p}-p\right)=0
$$

and one can see that it is automatically satisfied by the solution (4.28).

The expressions for $c\left(b_{i}, \mathfrak{n}_{a}\right)$ and $\mathcal{V}_{\text {on-shell }}\left(b_{i}, \mathfrak{n}_{a}\right)$ are too long to be reported here. One can explicitly verify that (3.16) and (3.19) are satisfied.

\section{$5 \quad \mathcal{I}$-extremization and black hole entropy}

As discussed in [12], the construction behind the gravity dual of $c$-extremization can be extended to twisted compactifications of three-dimensional CFTs on $\Sigma_{\mathfrak{g}}$. The gravity dual of the IR physics is a warped background $\mathrm{AdS}_{2} \times_{W} Y_{9}$ where $Y_{9}$ is topologically a fibration of a Sasaki-Einstein $Y_{7}$ over $\Sigma_{\mathfrak{g}}$. The gravity dual then describes the horizon of magnetically charged black holes in $\mathrm{AdS}_{4} \times Y_{7}$. As shown in [12] for the case of solutions of minimal gauged supergravity in four dimensions, the extremization of the analogue of the $c$-functional (3.12) reproduces the entropy of the black hole. It is then natural to conjecture that the construction in [12] is the dual of $\mathcal{I}$-extremization [21, 22], that it has been successfully used to perform a microscopic counting for $\mathrm{AdS}_{4}$ black holes. The $\mathcal{I}$ extremization principle states that the entropy of magnetically charged static black holes can be obtained by extremizing the logarithm of the supersymmetric partition function on $\Sigma_{\mathfrak{g}} \times S^{1}$ - also known as topologically twisted index. The index is a function of chemical potentials and magnetic fluxes for the global symmetries of the theory [47-49]. In the case 
of the ABJM theory [50], where $Y_{7}=S^{7}$, the $\mathcal{I}$-extremization principle states that the entropy of magnetically charged static black holes in $\mathrm{AdS}_{4} \times S^{7}$ is the extremum of the function $[21,22]$

$$
\mathcal{I}\left(\Delta_{a}, \mathfrak{n}_{a}\right)=-\frac{1}{2} \sum_{a=1}^{4} \mathfrak{n}_{a} \frac{\partial F_{S^{3}}\left(\Delta_{a}\right)}{\partial \Delta_{a}}
$$

where

$$
F_{S^{3}}\left(\Delta_{a}\right)=\frac{4 \pi \sqrt{2} N^{3 / 2}}{3} \sqrt{\Delta_{1} \Delta_{2} \Delta_{3} \Delta_{4}}
$$

and the chemical potentials and the fluxes satisfy the constraints

$$
\sum_{a=1}^{4} \Delta_{a}=2, \quad \sum_{a=1}^{4} \mathfrak{n}_{a}=2-2 \mathfrak{g} .
$$

As noticed in [20], the function (5.2) is the free energy on $S^{3}$ of ABJM.

In this note we show that the construction of [13] adapted to three-dimensional $\mathcal{N}=2$ theories, exactly reproduces (5.1) for ABJM and the identification is valid off-shell, when we use a natural parameterization of the R-charges in terms of the Reeb vector. The parallel with four dimensions is complete. As shown in $[18,19]$, the extremization of $F_{S^{3}}$ is equivalent to volume minimization for Calabi-Yau eight-folds [4, 5]. The equivalence of the construction of [13] with (5.1) is the analogue of (3.16).

It was shown in [20] that (5.1), where $\mathcal{I}$ is the logarithm of the topologically twisted index and $F_{S^{3}}\left(\Delta_{a}\right)$ the $S^{3}$ free energy, can be extended to many quivers dual to $\operatorname{AdS}_{4} \times Y_{7}$. (5.1) has been called the index theorem in [20]. This may suggest that the equivalence between the construction in [13] and $\mathcal{I}$-extremization extends to other Sasaki-Einstein manifolds. Since the equations are more complicated to solve for Calabi-Yau eight-folds we leave this very interesting investigation to future work. This might shed light on some puzzles about baryonic symmetries raised in [20, 23, 24].

\subsection{The ABJM theory}

Let us consider the twisted compactification of ABJM on $\Sigma_{\mathfrak{g}}$. The ABJM theory [50] is a three-dimensional supersymmetric Chern-Simons-matter theory with gauge group $\mathrm{U}(N)_{k} \times$ $\mathrm{U}(N)_{-k}$ (the subscripts denote the CS levels) with two bi-fundamental chiral fields $A_{i}$ in the representation $(\mathbf{N}, \overline{\mathbf{N}})$ and two bi-fundamental chiral fields $B_{i}$ in the representation $(\overline{\mathbf{N}}, \mathbf{N})$. The theory has a quartic superpotential

$$
W=\operatorname{Tr}\left(A_{1} B_{1} A_{2} B_{2}-A_{1} B_{2} A_{2} B_{1}\right) .
$$

We introduce four chemical potentials $\Delta_{a}$, one for each of the four fields $\left\{A_{i}, B_{i}\right\}$, and four fluxes $\mathfrak{n}_{a}$ on $\Sigma_{\mathfrak{g}}$ satisfying

$$
\sum_{a=1}^{4} \Delta_{a}=2, \quad \sum_{a=1}^{4} \mathfrak{n}_{a}=2-2 \mathfrak{g} .
$$


The ABJM theory in three dimensions is dual to $\operatorname{AdS}_{4} \times S^{7} / \mathbb{Z}_{k}$. We are interested in $k=1$, which corresponds to the toric Calabi-Yau four-fold $\mathbb{C}^{4}$. The toric data are

$$
v_{1}=(1,0,0,0), \quad v_{2}=(1,1,0,0), \quad v_{3}=(1,0,1,0), \quad v_{4}=(1,0,0,1) .
$$

We can associate each vertex to one of the fields. The supergravity background corresponding to the twisted compactification is then a warped background $\mathrm{AdS}_{2} \times_{W} Y_{9}$ where $Y_{9}$ is topologically a fibration of $S^{7}$ over $\Sigma_{\mathfrak{g}}$. It corresponds to the horizon geometry of the black holes found and studied in [51-53].

The master volume in [13] is defined as the volume of a dual polytope associated with the Kähler parameter $\lambda_{a}$ :

$$
\mathcal{V}=\frac{(2 \pi)^{4}}{|b|} \operatorname{Vol}\left(\left\{y \in H(b) \mid\left(y-y_{0}, v_{a}\right) \geq \lambda_{a}, a=1, \ldots, 4\right\}\right),
$$

where $H(b)$ is the hyperplane $(y, b)=1 / 2$ and $y_{0}=(1,0,0,0) /\left(2 b_{1}\right)$. Supersymmetry now requires $b_{1}=1$ [12]. For $\mathbb{C}^{4}$ the dual polytope is a tetrahedron lying on $H(b)$. Its four vertices can be found by solving for every distinct triple $v_{a}, v_{b}, v_{c}$ the equations

$$
\left(y-y_{0}, v_{a}\right)=\lambda_{a}, \quad\left(y-y_{0}, v_{b}\right)=\lambda_{b}, \quad\left(y-y_{0}, v_{c}\right)=\lambda_{c}, \quad\left(y-y_{0}, b\right)=0,
$$

and the volume can be easily computed. We then find that

$$
\mathcal{V}=\frac{8 \pi^{4}\left(\lambda_{1}\left(b_{2}+b_{3}+b_{4}-b_{1}\right)-\lambda_{2} b_{2}-\lambda_{3} b_{3}-\lambda_{4} b_{4}\right)^{3}}{3 b_{2} b_{3} b_{4}\left(b_{1}-b_{2}-b_{3}-b_{4}\right)}
$$

By adapting the arguments in [13], it is easy to see that equations (3.11) have the same form with the index $i$ running from 1 to 4 . Following [12, 13] we also define the entropy functional ${ }^{11}$

$$
S\left(b_{i}, \mathfrak{n}_{a}\right)=-\left.8 \pi^{2}\left(A \sum_{a=1}^{d} \frac{\partial \mathcal{V}}{\partial \lambda_{a}}+2 \pi b_{1} \sum_{i=1}^{4} n^{i} \frac{\partial \mathcal{V}}{\partial b_{i}}\right)\right|_{\lambda_{a}(b, \mathfrak{n}), A(b, \mathfrak{n})}
$$

The equations (3.11) are easily solved for the independent $\lambda_{a}$ and $A$. By substituting the result into (5.9) we find that

$$
\mathcal{V}_{\text {on-shell }}\left(b_{i}, \mathfrak{n}_{a}\right)=\frac{N^{3 / 2}}{6 \sqrt{2} b_{1} \pi^{2}} \sqrt{\frac{b_{2} b_{3} b_{4}\left(b_{1}-b_{2}-b_{3}-b_{4}\right)}{b_{1}}} .
$$

For the entropy functional we obtain

$$
S\left(b_{i}, \mathfrak{n}_{a}\right)=-\frac{2 \pi \sqrt{2} N^{3 / 2}}{3} \sqrt{\frac{b_{2} b_{3} b_{4}\left(b_{1}-b_{2}-b_{3}-b_{4}\right)}{b_{1}}}\left(\frac{\mathfrak{n}_{1}}{b_{1}-b_{2}-b_{3}-b_{4}}+\frac{\mathfrak{n}_{2}}{b_{2}}+\frac{\mathfrak{n}_{3}}{b_{3}}+\frac{\mathfrak{n}_{4}}{b_{4}}\right) .
$$

\footnotetext{
${ }^{11}$ In the notation of [12], we set $L^{6}=\left(2 \pi l_{P}\right)^{6}$.
} 
Similarly to what we did for $c$-extremization, we use the following parameterization for the R-charges

$$
\Delta_{a}\left(b_{i}, \mathfrak{n}_{a}\right)=-\left.\frac{2}{N} \frac{\partial \mathcal{V}}{\partial \lambda_{a}}\right|_{\lambda_{a}(b, \mathfrak{n}), A(b, \mathfrak{n})} .
$$

Plugging the solution to (3.11) into (5.13) we obtain

$$
\Delta_{1}\left(b_{i}\right)=\frac{2\left(b_{1}-b_{2}-b_{3}-b_{4}\right)}{b_{1}}, \quad \Delta_{2}\left(b_{i}\right)=\frac{2 b_{2}}{b_{1}}, \quad \Delta_{3}\left(b_{i}\right)=\frac{2 b_{3}}{b_{1}}, \quad \Delta_{4}\left(b_{i}\right)=\frac{2 b_{4}}{b_{1}} .
$$

As in $\mathcal{N}=4 \mathrm{SYM}$ in one dimension more (see (4.11)), the R-charges are functions of $b_{i}$ only. Moreover, one can also check that they are expressed in terms of the Sasaki volumes

$$
\Delta_{a}\left(b_{i}\right) \equiv \frac{2 \pi}{3 b_{1}} \frac{\operatorname{Vol}\left(S_{a}\right)}{\operatorname{Vol}\left(S^{7}\right)}
$$

of toric divisors (see $[4,5,19,54]$ for explicit expressions).

We now easily see that ${ }^{12}$

$$
\begin{aligned}
S\left(b_{i}, \mathfrak{n}_{a}\right)=\left.\mathcal{I}\left(\Delta_{a}, \mathfrak{n}_{a}\right)\right|_{\Delta_{a}(b)} & =-\left.\frac{2 \pi \sqrt{2} N^{3 / 2}}{3} \sum_{a=1}^{4} \mathfrak{n}_{a} \frac{\partial \sqrt{\Delta_{1} \Delta_{2} \Delta_{3} \Delta_{4}}}{\partial \Delta_{a}}\right|_{\Delta_{a}(b)} \\
& =-\left.\frac{1}{2} \sum_{a=1}^{4} \mathfrak{n}_{a} \frac{\partial F_{S^{3}}\left(\Delta_{a}\right)}{\partial \Delta_{a}}\right|_{\Delta_{a}(b)},
\end{aligned}
$$

thus proving that the functional $S\left(b_{i}, \mathfrak{n}_{a}\right)$ is equivalent to the $\mathcal{I}$-functional defined in $[21,22]$. It is also interesting to observe that

$$
\mathcal{V}_{\text {on-shell }}\left(b_{i}, \mathfrak{n}_{a}\right)=\left.\frac{1}{64 \pi^{3}} F_{S^{3}}\left(\Delta_{a}\right)\right|_{\Delta_{a}=\Delta_{a}\left(b_{i}\right)} .
$$

(5.16) and (5.17) are the direct analogues of (3.16) and (3.19).

The entropy of the black holes in [51-53] can be found equivalently by extremizing $S\left(b_{i}, \mathfrak{n}_{a}\right)$ with respect to $b_{2}, b_{3}, b_{4}$ after setting $b_{1}=1$ or by extremizing $\mathcal{I}\left(\Delta_{a}, \mathfrak{n}_{a}\right)$ with respect to $\Delta_{a}$ with the constraint $\sum_{a} \Delta_{a}=2$. More interestingly, the equivalence between the construction of [13] and the $\mathcal{I}$-extremization principle holds also off-shell. It would be interesting to see if this result extends to more general Sasaki-Einstein manifolds $Y_{7}$.

\section{Acknowledgments}

We would like to thank Noppadol Mekareeya for useful discussions. The work of SMH was supported by World Premier International Research Center Initiative (WPI Initiative), MEXT, Japan. AZ is partially supported by the INFN and ERC-STG grant 637844HBQFTNCER.

\footnotetext{
${ }^{12}$ We set $b_{1}=1$ in the following formulae. Reinstating $b_{1}$ we have $S=\sqrt{b_{1}} \mathcal{I}$ and $\mathcal{V}=\frac{\sqrt{b_{1}}}{64 \pi^{3}} F_{S^{3}}$.
} 


\section{A The relation between $c_{r}\left(\Delta_{a}, \mathfrak{n}_{a}\right)$ and $a\left(\Delta_{a}\right)$}

In this appendix we briefly review the derivation of (2.13) given in [17] and give an alternative one using the integration of the anomaly polynomial, in the spirit of appendix $\mathrm{C}$ of [41].

\section{A.1 Direct evaluation}

The trial a central charge of a four-dimensional $\mathcal{N}=1$ field theory with gauge group $G$, at large $N$, reads

$$
a\left(\Delta_{I}\right)=\frac{9}{32} \operatorname{Tr} R^{3}\left(\Delta_{I}\right)=\frac{9}{32}\left(\operatorname{dim} G+\sum_{I} \operatorname{dim} \mathfrak{R}_{I}\left(\Delta_{I}-1\right)^{3}\right),
$$

where the trace is taken over all the bi-fundamental fermions and gauginos and $\operatorname{dim} \mathfrak{R}_{I}$ is the dimension of the respective matter representation with R-charge $\Delta_{I}$. On the other hand, the trial right-moving central charge of the IR two-dimensional $\mathcal{N}=(0,2) \operatorname{SCFT}$ can be computed from the spectrum of massless fermions [2, 3, 42]. These are gauginos for all the gauge groups and fermionic zero modes for each chiral field. The difference between the number of fermions of opposite chiralities is predicted by the Riemann-Roch theorem and equals $\mathfrak{g}-1$ for gauginos and $-\mathfrak{n}_{I}-\mathfrak{g}+1$ for chiral fields $[2,3,42]$. The trial right-moving central charge is then given by

$$
c_{r}\left(\Delta_{I}, \mathfrak{n}_{I}\right)=3\left((\mathfrak{g}-1) \operatorname{dim} G+\sum_{I} \operatorname{dim} \mathfrak{R}_{I}\left(1-\mathfrak{n}_{I}-\mathfrak{g}\right)\left(\Delta_{I}-1\right)^{2}\right) .
$$

Using (A.1), it is easy to see that we can write

$$
c_{r}\left(\Delta_{I}, \mathfrak{n}_{I}\right)=-\frac{32}{9}(1-\mathfrak{g})\left(3 a\left(\Delta_{I}\right)+\sum_{I}\left(\frac{\mathfrak{n}_{I}}{1-\mathfrak{g}}-\Delta_{I}\right) \frac{\partial a\left(\Delta_{I}\right)}{\partial \Delta_{I}}\right) .
$$

When $a\left(\Delta_{I}\right)$ is a homogeneous function of degree three of the variables $\Delta_{I}$, (A.3) simplifies to

$$
c_{r}\left(\Delta_{I}, \mathfrak{n}_{I}\right)=-\frac{32}{9} \sum_{I} \mathfrak{n}_{I} \frac{\partial a\left(\Delta_{I}\right)}{\partial \Delta_{I}}
$$

which is precisely $(2.13)$.

In evaluating the right hand side of (A.3), we have considered the R-charges $\Delta_{I}$ of all the chiral fields as independent variables. However, the R-charges satisfy the constraint $\sum_{I \in W} \Delta_{I}=2$ for each term $W$ in the superpotential. Similarly $\sum_{I \in W} \mathfrak{n}_{I}=2-2 \mathfrak{g}$. Fortunately, the differential operator in (A.3) is such that we can impose the constraints equivalently before or after differentiation. (A.3) is indeed valid for all parameterizations of the R-charges and fluxes (even redundant ones) provided that, if we impose a constraint coming from a superpotential term $W, \sum_{I \in W} \Delta_{I}=2$, a similar constraint is imposed on $\mathfrak{n}_{I}, \sum_{I \in W} \mathfrak{n}_{I}=2-2 \mathfrak{g}$. In particular, it is valid for the parameterization used in this note, where we express $d-1$ independent R-charges in terms of $d$ parameters $\Delta_{a}$ with a constraint $\sum_{a=1}^{d} \Delta_{a}=2$. We can apply (A.3) and (A.4) considering $a$ as a function of $d$ independent variables $\Delta_{a}$ and impose the constraint $\sum_{a=1}^{d} \Delta_{a}=2$ after differentiation. 


\section{A.2 Integrating the anomaly polynomial}

The trial 't Hooft anomaly coefficients of two-dimensional $\mathcal{N}=(0,2)$ CFT can be extracted by integrating the six-form anomaly polynomial $I_{6}$ of the four-dimensional $\mathcal{N}=1$ field theory over $\Sigma_{\mathfrak{g}}[3,55-57]$. The six-form anomaly polynomial reads

$$
I_{6}=\frac{\operatorname{Tr}\left(F^{3}\right)}{6}-\frac{p_{1}(T \mathcal{M})}{24} \operatorname{Tr}(F),
$$

where $p_{1}(T \mathcal{M})$ is the first Poyntryagin class of tangent bundle, $F$ is the curvature of the Rand global symmetry bundle $K$ and the trace runs over all the fermions in the theory. We choose a basis of generators $T^{a}$ adapted to the parameterization discussed in section 2.1 and write $F=\sum_{a} \Delta_{a} T^{a} c_{1}(F)$, where $c_{1}(F)$ is a flux coupled to the U(1) R-symmetry and $\sum_{a} \Delta_{a}=2$. We can extract the trial $a$ central charge, at large $N$, from

$$
I_{6}=\frac{16}{27} a\left(\Delta_{a}\right) c_{1}(F)^{3},
$$

and we find

$$
a\left(\Delta_{a}\right)=\frac{9}{32} \sum_{a b c} c_{a b c} \Delta_{a} \Delta_{b} \Delta_{c},
$$

where $c_{a b c}=\operatorname{Tr}\left(T^{a} T^{b} T^{c}\right)$ are the t'Hooft anomaly coefficients.

Consider now the compactification of the four-dimensional theories on a Riemann surface $\Sigma_{\mathfrak{g}}$ with fluxes $\mathfrak{n}_{a}$. The prescription in $[3,55-57]$ for computing the anomaly coefficient $c_{r}$ of the two-dimensional SCFT amounts to first replace $F$ in (A.5) with

$$
F \rightarrow \sum_{a}\left(\Delta_{a} T^{a} c_{1}(F)-\frac{\mathfrak{n}_{a} T^{a}}{2-2 \mathfrak{g}} x\right),
$$

implementing the topological twist along $\Sigma_{\mathfrak{g}}$, and then integrate the $I_{6}$ on $\Sigma_{\mathfrak{g}}$ :

$$
I_{4}=\int_{\Sigma_{\mathfrak{g}}} I_{6}
$$

Here $x$ denotes the Chern root of the tangent bundle to $\Sigma_{\mathfrak{g}}, \Delta_{a}$ parameterize the trial R-symmetry, and $\mathfrak{n}_{a}$ are the fluxes parameterizing the twist, satisfying

$$
\sum_{a} \mathfrak{n}_{a}=2-2 \mathfrak{g}
$$

Then we integrate $I_{6}$ over $\Sigma_{\mathfrak{g}}$ using $\int_{\Sigma_{\mathfrak{g}}} x=2-2 \mathfrak{g}$. The result should be compared with the four-form anomaly polynomial of the two-dimensional SCFT that, in the large $N$ limit, where $c_{l}=c_{r}$, reads

$$
I_{4}=\frac{c_{r}\left(\Delta_{a}, \mathfrak{n}_{a}\right)}{6} c_{1}(F)^{2} .
$$

We see immediately that, since in our basis $a\left(\Delta_{a}\right)$ is homogeneous,

$$
c_{r}\left(\Delta_{a}, \mathfrak{n}_{a}\right)=-\frac{32}{9} \sum_{a} \mathfrak{n}_{a} \frac{\partial a\left(\Delta_{a}\right)}{\partial \Delta_{a}},
$$

which is precisely $(2.13)$. 


\section{B Proof of the equality between $c\left(b_{i}, \mathfrak{n}_{a}\right)$ and $c_{r}\left(\Delta_{a}, \mathfrak{n}_{a}\right)$}

In this appendix we prove (3.18). As discussed in the text, (3.16) and (3.17) are simple consequences of this equation. We will also solve explicitly the equations (3.11) in a particular gauge. We first review in appendix B.1 some technical results of [13] that will be used in the rest of the proof. For simplicity of notations, in this appendix $\Delta_{a}$ will always refer to the quantities $\Delta_{a}\left(b_{i}, \mathfrak{n}_{a}\right)$ defined in (3.14), unless otherwise stated.

\section{B.1 Some simplifications}

The master volume is a quadratic form in $\lambda_{a},{ }^{13}$

$$
\mathcal{V}=\frac{1}{2} \sum_{a, b=1}^{d} J_{a b} \lambda_{a} \lambda_{b}
$$

with a symmetric matrix $J_{a b}$, of rank $d-2$. Indeed, it is invariant under

$$
\lambda_{a} \rightarrow \lambda_{a}+\sum_{i=1}^{3} l_{i}\left(b_{1} v_{a}^{i}-b_{i}\right),
$$

since, using $[13,(3.41)]$, we find that

$$
\delta_{l_{i}} \mathcal{V}=\sum_{a=1}^{d} \frac{\partial \mathcal{V}}{\partial \lambda_{a}} \delta_{l_{i}} \lambda_{a}=\sum_{a=1}^{d} \frac{\partial \mathcal{V}}{\partial \lambda_{a}}\left(b_{1} v_{a}^{i}-b_{i}\right)=0
$$

Notice that this leaves $d-2$ independent $\lambda_{a}$ since $l_{1}$ does not contribute $\left(v_{a}^{1}=1\right.$ for all $\left.a\right)$. Correspondingly, the matrix $J_{a, a}$ has rank $d-2$. Since (B.3) is valid for all $b_{i}$ and all $\lambda_{a}$ we obtain

$$
\sum_{a=1}^{d} \frac{\partial \mathcal{V}}{\partial \lambda_{a}}\left(b_{1} v_{a}^{i}-b_{i}\right)=\sum_{a, b=1}^{d} J_{a b} \lambda_{b}\left(b_{1} v_{a}^{i}-b_{i}\right)=0 \Longrightarrow \sum_{a=1}^{d} v_{a}^{i} J_{a b}=\frac{b_{i}}{b_{1}} \sum_{a=1}^{d} J_{a b},
$$

The equations (3.11) can be written as

$$
\begin{aligned}
N & =-\sum_{a, b} J_{a b} \lambda_{b}, \\
\mathfrak{n}_{a} N & =-\frac{A}{2 \pi} \sum_{b} J_{a b}-b_{1} \sum_{i, b} n^{i} \frac{\partial J_{a b}}{\partial b_{i}} \lambda_{b}, \\
A \sum_{a b} J_{a b} & =2 \pi n^{1} \sum_{a b} J_{a b} \lambda_{b}-2 \pi b_{1} \sum_{a, b, i} n^{i} \frac{\partial J_{a b}}{\partial b_{i}} \lambda_{b} .
\end{aligned}
$$

For given fluxes $\mathfrak{n}_{a}$ and number of colors $N$, these are, in principle, $d+2$ equations for $d-1=(d-2)+1$ variables $\lambda_{a}$ and $A$. But, fortunately, three equations are redundant [13]. Indeed the three linear combinations, $k=1,2,3$, of the equations for $\mathfrak{n}_{a}$

$$
\sum_{a} v_{a}^{k} \mathfrak{n}_{a}=-\frac{1}{2 \pi N} \frac{b_{k}}{b_{1}}\left(A \sum_{a b} J_{a b}-2 \pi n^{1} \sum_{a b} J_{a b} \lambda_{b}+2 \pi b_{1} \sum_{a, b, i} n^{i} \frac{\partial J_{a b}}{\partial b_{i}} \lambda_{b}\right)-\frac{n^{k}}{N} \sum_{a b} J_{a b} \lambda_{b} \equiv n^{k}
$$

\footnotetext{
${ }^{13}$ To compare with [13]: $J_{a b}=(2 \pi)^{2} I_{a b}$.
} 
reproduce the relation between $\mathfrak{n}_{a}$ and $n^{k}$. We used the first and third equations in (3.11), the constraint (B.4) and its derivative with respect to $b_{i}$

$$
\sum_{a} v_{a}^{k} \frac{\partial J_{a b}}{\partial b_{i}}=\frac{b_{k}}{b_{1}} \sum_{a} \frac{\partial J_{a b}}{\partial b_{i}}+\left(\delta^{i k} \frac{1}{b_{1}}-\delta^{i 1} \frac{b_{k}}{\left(b_{1}\right)^{2}}\right) \sum_{a} J_{a b}
$$

We can also rewrite the functional (3.12) as

$$
c\left(b_{i}, \mathfrak{n}_{a}\right)=-48 \pi^{2}\left(A \sum_{a, b} J_{a b} \lambda_{b}+\pi b_{1} \sum_{a, b, i} n^{i} \frac{\partial J_{a b}}{\partial b_{i}} \lambda_{a} \lambda_{b}\right)=48 \pi^{2} N\left(\frac{A}{2}+\pi \sum_{a=1}^{d} \lambda_{a} \mathfrak{n}_{a}\right),
$$

where, in the second step, we computed $\sum_{a} \lambda_{a} \mathfrak{n}_{a}$ from the second equation in (B.5).

The R-charges (3.14) read

$$
\Delta_{a}=-\frac{2}{N} \frac{\partial \mathcal{V}}{\partial \lambda_{a}}=-\frac{2}{N} \sum_{b} J_{a b} \lambda_{b}
$$

Multiplying (B.4) by $-\frac{2}{N} \lambda_{b}$ and summing over $b$ we obtain

$$
\sum_{a=1}^{d} v_{a}^{i} \Delta_{a}=-\frac{2}{N} \frac{b_{i}}{b_{1}} \sum_{a, b=1}^{d} J_{a b} \lambda_{b}=2 \frac{b_{i}}{b_{1}}
$$

Notice, in particular, that $\sum_{a} \Delta_{a}=2$. Introducing the vectors $r_{a}=v_{a}-b / b_{1}$ we can also write

$$
\sum_{a=1}^{d} r_{a} \Delta_{a}=0
$$

an identity that we will use repeatedly in the following.

\section{B.2 A convenient gauge}

We can simplify the equations choosing a gauge. Using (B.2) we can set two $\lambda_{a}$ to zero, say $\lambda_{1}=\lambda_{2}=0$. From (3.10), we see that the non-zero components of the matrix $J_{a b}$ are

$$
J_{a, a+1}=8 \pi^{3} \frac{1}{\left(v_{a}, v_{a+1}, b\right)}, \quad J_{a, a}=-8 \pi^{3} \frac{\left(v_{a-1}, v_{a+1}, b\right)}{\left(v_{a-1}, v_{a}, b\right)\left(v_{a}, v_{a+1}, b\right)} .
$$

We also know that

$$
\Delta_{a}=-\frac{2}{N} \sum_{b=1}^{d} J_{a b} \lambda_{b}
$$

In our gauge, $\lambda_{1}=\lambda_{2}=0$, we find that

$$
\begin{aligned}
& \Delta_{2}=-\frac{2}{N}\left(J_{23} \lambda_{3}\right), \\
& \Delta_{3}=-\frac{2}{N}\left(J_{33} \lambda_{3}+J_{34} \lambda_{4}\right), \\
& \Delta_{4}=-\frac{2}{N}\left(J_{43} \lambda_{3}+J_{44} \lambda_{4}+J_{45} \lambda_{5}\right),
\end{aligned}
$$


that we can solve recursively. We obtain

$$
\begin{aligned}
\lambda_{3} & =-\frac{N}{16 \pi^{3}}\left(v_{2}, v_{3}, b\right) \Delta_{2}, \\
\lambda_{4} & =-\frac{N}{16 \pi^{3}}\left(\left(v_{2}, v_{4}, b\right) \Delta_{2}+\left(v_{3}, v_{4}, b\right) \Delta_{3}\right), \\
\lambda_{5} & =-\frac{N}{16 \pi^{3}}\left(v_{4}, v_{5}, b\right)\left(\Delta_{4}+\frac{\left(v_{3}, v_{5}, b\right)}{\left(v_{4}, v_{5}, b\right)} \Delta_{3}+\frac{\left(v_{2}, v_{4}, b\right)\left(v_{3}, v_{5}, b\right)-\left(v_{2}, v_{3}, b\right)\left(v_{4}, v_{5}, b\right)}{\left(v_{3}, v_{4}, b\right)\left(v_{4}, v_{5}, b\right)} \Delta_{2}\right) \\
& =-\frac{N}{16 \pi^{3}}\left(\left(v_{4}, v_{5}, b\right) \Delta_{4}+\left(v_{3}, v_{5}, b\right) \Delta_{3}+\left(v_{2}, v_{5}, b\right) \Delta_{2}\right),
\end{aligned}
$$

where in the last step we used the identity

$$
(A, B, b)(C, D, b)-(A, C, b)(B, D, b)-(A, D, b)(C, B, b)=0,
$$

valid for arbitrary vectors $A, B, C, D$. Altogether we can write the inversion formula

$$
\lambda_{a}=-\frac{N}{16 \pi^{3}} \sum_{c=2}^{a}\left(v_{c}, v_{a}, b\right) \Delta_{c}, \quad a=3, \ldots, d
$$

As a consistency check, note that we can also extract $\lambda_{d}$ from the equation $\Delta_{1}=-\frac{2}{N} J_{d 1} \lambda_{d}$, obtaining

$$
\lambda_{d}=-\frac{N}{16 \pi^{3}}\left(v_{d}, v_{1}, b\right) \Delta_{1} \equiv-\frac{N}{16 \pi^{3}} \sum_{c=2}^{d}\left(v_{c}, v_{d}, b\right) \Delta_{c},
$$

where in the second step we used $\sum_{c} v_{c} \Delta_{c}=2 b / b_{1}$.

We can now analyze the equations (B.5) in the gauge $\lambda_{1}=\lambda_{2}=0$. Introduce the notation $\nabla \equiv \sum_{i} n^{i} \partial_{b_{i}}$. We have

$$
\nabla J_{a, a+1}=-8 \pi^{3} \frac{\left(v_{a}, v_{a+1}, n\right)}{\left(v_{a}, v_{a+1}, b\right)^{2}}, \quad \sum_{b} J_{a b}=8 \pi^{3} b_{1} \frac{\left(v_{a-1}, v_{a}, v_{a+1}\right)}{\left(v_{a-1}, v_{a}, b\right)\left(v_{a}, v_{a+1}, b\right)},
$$

where, for the second identity, we used ${ }^{14}$

$$
\left(v_{a-1}, v_{a}, b\right)+\left(v_{a}, v_{a+1}, b\right)-\left(v_{a-1}, v_{a+1}, b\right)=b_{1}\left(v_{a-1}, v_{a}, v_{a+1}\right) .
$$

Writing the equations (B.5) for $a=1$ and $a=2$

$$
\begin{aligned}
& -N \mathfrak{n}_{2}=\frac{A}{2 \pi}\left(J_{12}+J_{22}+J_{23}\right)+b_{1} \nabla J_{23} \lambda_{3}, \\
& -N \mathfrak{n}_{1}=\frac{A}{2 \pi}\left(J_{1 d}+J_{11}+J_{12}\right)+b_{1} \nabla J_{1 d} \lambda_{d},
\end{aligned}
$$

\footnotetext{
${ }^{14}$ The geometrical meaning of this identity is clear from figure (B.26): the sum of the areas of the plane triangles of vertices $\left(v_{a-1}, v_{a}, B\right)$ and $\left(v_{a}, v_{a+1}, B\right)$ minus the area of $\left(v_{a-1}, v_{a+1}, B\right)$ is the area of $\left(v_{a-1}, v_{a}, v_{a+1}\right)$. The factor of $b_{1}$ takes into account the normalization in converting three-dimensional determinants into areas in the plane.
} 
and using (B.15) and (B.18) we find

$$
\begin{aligned}
& \frac{N b_{1}}{16 \pi^{3}}\left(v_{2}, v_{3}, n\right) \Delta_{2}=-\frac{A}{2 \pi} b_{1} \frac{\left(v_{1}, v_{2}, v_{3}\right)}{\left(v_{1}, v_{2}, b\right)}-\frac{\left(v_{2}, v_{3}, b\right)}{8 \pi^{3}} N \mathfrak{n}_{2}, \\
& \frac{N b_{1}}{16 \pi^{3}}\left(v_{d}, v_{1}, n\right) \Delta_{1}=-\frac{A}{2 \pi} b_{1} \frac{\left(v_{d}, v_{1}, v_{2}\right)}{\left(v_{1}, v_{2}, b\right)}-\frac{\left(v_{d}, v_{1}, b\right)}{8 \pi^{3}} N \mathfrak{n}_{1} .
\end{aligned}
$$

Multiplying the first by $\left(v_{d}, v_{1}, v_{2}\right)$ and the second by $\left(v_{1}, v_{2}, v_{3}\right)$ and subtracting we obtain

$$
\begin{aligned}
& \left(v_{d}, v_{1}, v_{2}\right)\left(v_{2}, v_{3}, n\right) \Delta_{2}-\left(v_{1}, v_{2}, v_{3}\right)\left(v_{d}, v_{1}, n\right) \Delta_{1} \\
& =-\frac{2}{b_{1}}\left(\left(v_{d}, v_{1}, v_{2}\right)\left(v_{2}, v_{3}, b\right) \mathfrak{n}_{2}-\left(v_{1}, v_{2}, v_{3}\right)\left(v_{d}, v_{1}, b\right) \mathfrak{n}_{1}\right) .
\end{aligned}
$$

This has been proved for $a=1$ and $a=2$ but should hold for all adjacent pairs $(a, a+1)$ because it is an identity for gauge invariant quantities and we can always use an adapted gauge where $\lambda_{a}=\lambda_{a+1}=0$. Therefore, we find

$$
\begin{aligned}
& \left(v_{a-1}, v_{a}, v_{a+1}\right)\left(v_{a+1}, v_{a+2}, n\right) \Delta_{a+1}-\left(v_{a}, v_{a+1}, v_{a+2}\right)\left(v_{a-1}, v_{a}, n\right) \Delta_{a} \\
& =-\frac{2}{b_{1}}\left(\left(v_{a-1}, v_{a}, v_{a+1}\right)\left(v_{a+1}, v_{a+2}, b\right) \mathfrak{n}_{a+1}-\left(v_{a}, v_{a+1}, v_{a+2}\right)\left(v_{a-1}, v_{a}, b\right) \mathfrak{n}_{a}\right)
\end{aligned}
$$

where we identify $\mathfrak{n}_{a+d}=\mathfrak{n}_{a}, \Delta_{a+d}=\Delta_{a}$ and $v_{a+d}=v_{a}$, so that for example $v_{d+1}=v_{1}$ and $v_{0}=v_{d}$. This is a set of equations that allow to find an explicit expression for $\Delta_{a}$ using recursion to obtain $\Delta_{a+1}$ from $\Delta_{a}$ and enforcing $\sum_{a} \Delta_{a}=2$ in order to find the value of $\Delta_{1}$. Notice that, at each step of the recursion, $b$ only appears linearly so $\Delta_{a}$ is a regular function of $b_{i} . \Delta_{a}$ is actually a linear polynomial in $b / b_{1}$.

For further reference let us also quote the value of $A$ :

$$
A=-\frac{N}{8 \pi^{2}} \frac{\left(v_{2}, v_{3}, n\right)\left(v_{1}, v_{2}, b\right)}{\left(v_{1}, v_{2}, v_{3}\right)} \Delta_{2}-\frac{N}{4 \pi^{2} b_{1}} \frac{\left(v_{2}, v_{3}, b\right)\left(v_{1}, v_{2}, b\right)}{\left(v_{1}, v_{2}, v_{3}\right)} \mathfrak{n}_{2}
$$

This equation and (B.17) guarantee that, in the gauge $\lambda_{1}=\lambda_{2}=0, \lambda_{a} / b_{1}$ and $A / b_{1}$ are quadratic polynomials in $b / b_{1}$.

\section{B.3 Completing the proof}

We now prove (3.18) using the logic of [9] and [10]. In figure (B.26) we draw the plane orthogonal to the vector $e_{1}=(1,0,0)$, where all the endpoints of the vectors $v_{a}$ lie. The vectors $w_{a}=v_{a+1}-v_{a}$ lie entirely on the plane and correspond to the sides of the toric diagram. We also define the vectors $r_{a}=v_{a}-b / b_{1}$. They also lie entirely on the plane and connect the point $B$ with coordinates $\left(b_{2} / b_{1}, b_{3} / b_{2}\right)$ to the vertices of the toric diagram. All the vectors in the following are three-dimensional. We use $\langle C, D\rangle=\left(e_{1}, C, D\right)$ to compute areas in the plane. Indeed, when $C$ and $D$ are vectors lying on the plane, $|\langle C, D\rangle|$ is twice the area of the triangle with sides $C$ and $D$. We also assume that the vertices are labeled 
in the counterclockwise direction.

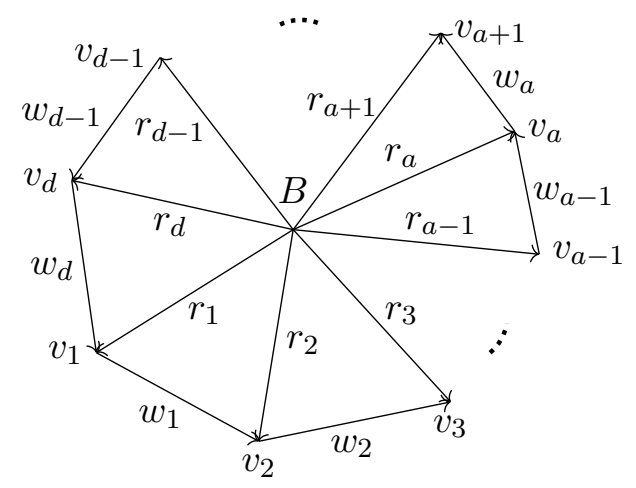

Define the quantities

$$
c_{a}=\sum_{b, c=1}^{d}\left|\left(v_{a}, v_{b}, v_{c}\right)\right| \mathfrak{n}_{b} \Delta_{c}=\sum_{b, c \in[a+1, a-1]}\left(v_{a}, v_{b}, v_{c}\right)\left(\mathfrak{n}_{b} \Delta_{c}+\mathfrak{n}_{c} \Delta_{b}\right),
$$

where the symbol $b, c \in[A, B]$ means a sum over all pairs $A \leq b<c \leq B+d$ with the identification $\mathfrak{n}_{a+d}=\mathfrak{n}_{a}, \Delta_{a+d}=\Delta_{a}$ and $v_{a+d}=v_{a}$. If we select an order, we can drop the absolute value from $\left|\left(v_{a}, v_{b}, v_{c}\right)\right|$ but note that we need to keep the symmetrized product of $\mathfrak{n}$ and $\Delta$. We want to prove that there exists a function $S$ and a vector $u$ such that

$$
c_{a}=S+\left\langle r_{a}, u\right\rangle,
$$

and $S$ is proportional to $c\left(b_{i}, \mathfrak{n}_{a}\right)$. Using repeatedly the identity $\left(v_{a}, v_{b}, v_{c}\right)=\left\langle r_{a}, r_{b}\right\rangle+$ $\left\langle r_{b}, r_{c}\right\rangle-\left\langle r_{a}, r_{c}\right\rangle,{ }^{15}$ we can compute the difference

$$
\begin{aligned}
c_{2}-c_{1}= & \sum_{b, c \in[3, d]}\left(v_{2}-v_{1}, v_{b}, v_{c}\right)\left(\mathfrak{n}_{b} \Delta_{c}+\mathfrak{n}_{c} \Delta_{b}\right)+\sum_{b \in[3, d]}\left(v_{2}, v_{b}, v_{1}\right)\left(\mathfrak{n}_{b} \Delta_{1}+\mathfrak{n}_{1} \Delta_{b}\right) \\
& -\sum_{c \in[3, d]}\left(v_{1}, v_{2}, v_{c}\right)\left(\mathfrak{n}_{2} \Delta_{c}+\mathfrak{n}_{c} \Delta_{2}\right)=\sum_{b, c \in[3, d]}\left\langle w_{1},\left(r_{b}-r_{c}\right)\left(\mathfrak{n}_{b} \Delta_{c}+\mathfrak{n}_{c} \Delta_{b}\right)\right\rangle \\
& +\sum_{b \in[3, d]}\left\langle w_{1},\left(r_{b}-r_{1}\right)\left(\mathfrak{n}_{b} \Delta_{1}+\mathfrak{n}_{1} \Delta_{b}\right)\right\rangle+\sum_{c \in[3, d]}\left\langle w_{1},\left(r_{2}-r_{c}\right)\left(\mathfrak{n}_{2} \Delta_{c}+\mathfrak{n}_{c} \Delta_{2}\right)\right\rangle \\
= & \sum_{b, c \in[2,1]}\left\langle w_{1},\left(r_{b}-r_{c}\right)\left(\mathfrak{n}_{b} \Delta_{c}+\mathfrak{n}_{c} \Delta_{b}\right)+d_{1} w_{1}\right\rangle \equiv\left\langle w_{1}, u_{1}\right\rangle,
\end{aligned}
$$

where we added an arbitrary term $d_{1} w_{1}$ since it gives a vanishing contribution. More generally we find

$$
c_{a+1}-c_{a}=\left\langle w_{a}, u_{a}\right\rangle \equiv\left\langle w_{a}, \sum_{b, c \in[a+1, a]}\left(r_{b}-r_{c}\right)\left(\mathfrak{n}_{b} \Delta_{c}+\mathfrak{n}_{c} \Delta_{b}\right)+d_{a} w_{a}\right\rangle,
$$

\footnotetext{
${ }^{15}$ The geometrical interpretation of this identity is similar to the one discussed in footnote 14 .
} 
where again $d_{a}$ is arbitrary. We will show now that there exists a choice of $d_{a}$ such that all the $u_{a}$ are equal. In order to show it, we compute the difference

$$
\begin{aligned}
u_{2}-u_{1}= & \sum_{b, c \in[3,2]}\left(r_{b}-r_{c}\right)\left(\mathfrak{n}_{b} \Delta_{c}+\mathfrak{n}_{c} \Delta_{b}\right)-\sum_{b, c \in[2,1]}\left(r_{b}-r_{c}\right)\left(\mathfrak{n}_{b} \Delta_{c}+\mathfrak{n}_{c} \Delta_{b}\right)+d_{2} w_{2}-d_{1} w_{1} \\
= & \sum_{a \in[3, d]}\left[\left(r_{a}-r_{1}\right)\left(\mathfrak{n}_{a} \Delta_{1}+\mathfrak{n}_{1} \Delta_{a}\right)+\left(r_{a}-r_{2}\right)\left(\mathfrak{n}_{a} \Delta_{2}+\mathfrak{n}_{2} \Delta_{a}\right)\right]+\left(r_{1}-r_{2}\right)\left(\mathfrak{n}_{1} \Delta_{2}+\mathfrak{n}_{2} \Delta_{1}\right) \\
& -\sum_{a \in[3, d]}\left[\left(r_{a}-r_{1}\right)\left(\mathfrak{n}_{a} \Delta_{1}+\mathfrak{n}_{1} \Delta_{a}\right)+\left(r_{2}-r_{a}\right)\left(\mathfrak{n}_{2} \Delta_{a}+\mathfrak{n}_{a} \Delta_{2}\right)\right]+\left(r_{2}-r_{1}\right)\left(\mathfrak{n}_{2} \Delta_{1}+\mathfrak{n}_{1} \Delta_{2}\right) \\
& +d_{2} w_{2}-d_{1} w_{1}=-2 \sum_{a \in[3,1]}\left(r_{2}-r_{a}\right)\left(\mathfrak{n}_{a} \Delta_{2}+\mathfrak{n}_{2} \Delta_{a}\right)+d_{2} w_{2}-d_{1} w_{1} \\
= & -2\left[r_{2}\left(\Delta_{2} \sum_{a} \mathfrak{n}_{a}+2 \mathfrak{n}_{2}\right)-\Delta_{2}\left(\sum_{a} r_{a} \mathfrak{n}_{a}\right)\right]+d_{2} w_{2}-d_{1} w_{1},
\end{aligned}
$$

where we used $\sum_{b=1}^{d} \Delta_{b}=2$ and $\sum_{a=1}^{d} r_{a} \Delta_{a}=0$. More generally,

$$
u_{a+1}-u_{a}=-2\left[r_{a+1}\left(\Delta_{a+1} \sum_{b} \mathfrak{n}_{b}+2 \mathfrak{n}_{a+1}\right)-\Delta_{a+1} \sum_{b} r_{b} \mathfrak{n}_{b}\right]+d_{a+1} w_{a+1}-d_{a} w_{a}
$$

Requiring that (B.32) is zero gives an expression for $d_{a}$. Indeed, if $u_{a+1}=u_{a}$, contracting (B.32) with $w_{a}$ and $w_{a+1}$, respectively, we obtain two different expressions for $d_{a}$ :

$$
\begin{aligned}
& d_{a+1}\left\langle w_{a}, w_{a+1}\right\rangle=2 \Delta_{a+1}\left(\left\langle w_{a}, r_{a+1}\right\rangle \sum_{b} \mathfrak{n}_{b}-\sum_{b} \mathfrak{n}_{b}\left\langle w_{a}, r_{b}\right\rangle\right)+4 \mathfrak{n}_{a+1}\left\langle w_{a}, r_{a+1}\right\rangle \\
& d_{a}\left\langle w_{a}, w_{a+1}\right\rangle=2 \Delta_{a+1}\left(\left\langle w_{a+1}, r_{a+1}\right\rangle \sum_{b} \mathfrak{n}_{b}-\sum_{b} \mathfrak{n}_{b}\left\langle w_{a+1}, r_{b}\right\rangle\right)+4 \mathfrak{n}_{a+1}\left\langle w_{a+1}, r_{a+1}\right\rangle .
\end{aligned}
$$

The two expressions should coincide. By shifting $a \rightarrow a+1$ in the second equation and comparing with the first one, we get the consistency condition

$$
\begin{aligned}
& \Delta_{a+2}\left\langle w_{a}, w_{a+1}\right\rangle\left(\left\langle w_{a+2}, r_{a+2}\right\rangle \sum_{b} \mathfrak{n}_{b}-\sum_{b} \mathfrak{n}_{b}\left\langle w_{a+2}, r_{b}\right\rangle\right)+2 \mathfrak{n}_{a+2}\left\langle w_{a}, w_{a+1}\right\rangle\left\langle w_{a+2}, r_{a+2}\right\rangle \\
& -\Delta_{a+1}\left\langle w_{a+1}, w_{a+2}\right\rangle\left(\left\langle w_{a}, r_{a+1}\right\rangle \sum_{b} \mathfrak{n}_{b}-\sum_{b} \mathfrak{n}_{b}\left\langle w_{a}, r_{b}\right\rangle\right)-2 \mathfrak{n}_{a+1}\left\langle w_{a+1}, w_{a+2}\right\rangle\left\langle w_{a}, r_{a+1}\right\rangle=0 .
\end{aligned}
$$

After a short computation using $\sum_{a} \mathfrak{n}_{a} v_{a}=n$ and some geometrical identities to convert areas in the plane to three-dimensional determinants, like

$$
\begin{aligned}
& \left\langle w_{a+2}, r_{a+2}\right\rangle \sum_{b} \mathfrak{n}_{b}-\sum_{b} \mathfrak{n}_{b}\left\langle w_{a+2}, r_{b}\right\rangle=\left\langle w_{a+2}, n^{1} v_{a+2}-n\right\rangle=-\left(v_{a+2}, v_{a+3}, n\right), \\
& \left\langle w_{a}, w_{a+1}\right\rangle=\left(v_{a}, v_{a+1}, v_{a+2}\right), \quad\left\langle w_{a}, r_{a}\right\rangle=-\frac{1}{b_{1}}\left(v_{a}, v_{a+1}, b\right),
\end{aligned}
$$


and generalizations, we obtain

$$
\begin{aligned}
& \Delta_{a+2}\left(v_{a}, v_{a+1}, a_{a+2}\right)\left(v_{a+2}, v_{a+3}, n\right)-\Delta_{a+1}\left(v_{a+1}, v_{a+2}, v_{a+3}\right)\left(v_{a}, v_{a+1}, n\right)= \\
& -\frac{2}{b_{1}}\left(\mathfrak{n}_{a+2}\left(v_{a}, v_{a+1}, v_{a+2}\right)\left(v_{a+2}, v_{a+3}, b\right)-\mathfrak{n}_{a+1}\left(v_{a+1}, v_{a+2}, v_{a+3}\right)\left(v_{a}, v_{a+1}, b\right)\right),
\end{aligned}
$$

which is precisely the identity (B.24). This proves that $d_{a}$ such that all the $u_{a}$ are equal can be actually found. We can also write an explicit expression

$$
d_{a}=-\frac{2 \Delta_{a+1} b_{1}\left(v_{a+1}, v_{a+2}, n\right)+4 \mathfrak{n}_{a+1}\left(v_{a+1}, v_{a+2}, b\right)}{b_{1}\left(v_{a}, v_{a+1}, v_{a+2}\right)} .
$$

Notice also that, comparing with (B.25), we obtain

$$
A=\frac{N}{16 \pi^{2}}\left(v_{1}, v_{2}, b\right) d_{1} .
$$

Since all the vectors $u_{a}$ are equal we call them $u$. We have

$$
c_{a+1}-c_{a}=\left\langle w_{a}, u\right\rangle=\left\langle v_{a+1}-v_{a}, u\right\rangle \Longrightarrow c_{a}=S+\left\langle r_{a}, u\right\rangle,
$$

for some function $S$. Using (B.27) and (B.30), we find that

$$
\begin{aligned}
S=c_{1}-\left\langle r_{1}, u\right\rangle= & \sum_{b, c \in[2, d]}\left(\left\langle r_{1}, r_{b}\right\rangle+\left\langle r_{b}, r_{c}\right\rangle-\left\langle r_{1}, r_{c}\right\rangle\right)\left(\mathfrak{n}_{b} \Delta_{c}+\mathfrak{n}_{c} \Delta_{b}\right) \\
& -\sum_{b, c \in[2,1]}\left(\left\langle r_{1}, r_{b}\right\rangle-\left\langle r_{1}, r_{c}\right\rangle\right)\left(\mathfrak{n}_{b} \Delta_{c}+\mathfrak{n}_{c} \Delta_{b}\right)-d_{1}\left\langle r_{1}, w_{1}\right\rangle \\
= & \sum_{b, c \in[2,1]}\left\langle r_{b}, r_{c}\right\rangle\left(\mathfrak{n}_{b} \Delta_{c}+\mathfrak{n}_{c} \Delta_{b}\right)-\frac{16 \pi^{2} A}{b_{1} N} .
\end{aligned}
$$

We can manipulate the sum in the previous expression by using repeatedly $\sum_{c} \Delta_{c} r_{c}=0$ :

$$
\begin{aligned}
& \sum_{b, c \in[2,1]}\left\langle r_{b}, r_{c}\right\rangle \mathfrak{n}_{b} \Delta_{c}+\sum_{b, c \in[2,1]}\left\langle r_{b}, r_{c}\right\rangle \mathfrak{n}_{c} \Delta_{b}=\sum_{c, b \in[2,1]}\left\langle r_{c}, r_{b}\right\rangle \mathfrak{n}_{c} \Delta_{b}+\sum_{b, c \in[2, d]}\left\langle r_{b}, r_{c}\right\rangle \mathfrak{n}_{c} \Delta_{b} \\
& =-\sum_{b, c \in[2, d]}\left\langle r_{c}, r_{b}\right\rangle \mathfrak{n}_{c} \Delta_{b}+\sum_{b, c \in[2, d]}\left\langle r_{b}, r_{c}\right\rangle \mathfrak{n}_{c} \Delta_{b}=2 \sum_{b, c \in[2, d]}\left\langle r_{b}, r_{c}\right\rangle \mathfrak{n}_{c} \Delta_{b},
\end{aligned}
$$

where in the first step we change variables in the first sum and notice that the term $c=1$ in the second sum is zero because of $\sum_{c} r_{c} \Delta_{c}=0$. In the second step, for each fixed $c$, we transform $\sum_{c<b \leq 1} r_{b} \Delta_{b}=-\sum_{2 \leq b \leq c} r_{b} \Delta_{b}$ and notice that $c \leq d$. In conclusion we have

$$
S=2 \sum_{b, c \in[2, d]}\left\langle r_{b}, r_{c}\right\rangle \mathfrak{n}_{c} \Delta_{b}-\frac{16 \pi^{2} A}{b_{1} N} .
$$

This has to be compared with (B.8):

$$
c_{r}=48 \pi^{2} N\left(\frac{A}{2}-\frac{N b_{1}}{16 \pi^{2}} \sum_{a=3}^{d} \sum_{c=2}^{a}\left\langle r_{c}, r_{a}\right\rangle \Delta_{c} \mathfrak{n}_{a}\right)=48 \pi^{2} N\left(\frac{A}{2}-\frac{N b_{1}}{16 \pi^{2}} \sum_{b, c \in[2, d]}\left\langle r_{b}, r_{c}\right\rangle \mathfrak{n}_{c} \Delta_{b}\right),
$$


where we worked in the gauge $\lambda_{1}=\lambda_{2}=0$ and we used (B.17) and the identity $\left(v_{c}, v_{a}, b\right)=$ $b_{1}\left\langle r_{c}, r_{a}\right\rangle$. We see that

$$
S=-\frac{2}{3 N^{2} b_{1}} c_{r}
$$

Multiplying (B.28) by $-3 N^{2}$, and using the definition (B.27) we finally obtain

$$
-6 \sum_{b, c=1}^{d} c_{a b c} \mathfrak{n}_{b} \Delta_{c}=-3 N^{2} \sum_{b, c=1}^{d}\left|\left(v_{a}, v_{b}, v_{c}\right)\right| \mathfrak{n}_{b} \Delta_{c}=c_{r}+\left(e_{1}, r_{a}, t\right),
$$

where we set $b_{1}=2$ and $t=-3 N^{2} u$. This is exactly (3.18).

\section{B.4 Proving that $\mathcal{V}_{\text {on-shell }}\left(b_{i}, \mathfrak{n}_{a}\right)=a\left(\Delta_{a}\right)$}

In the gauge $\lambda_{1}=\lambda_{2}=0$, the master volume (3.10) can be written as

$$
\begin{aligned}
\mathcal{V} & =-\frac{N}{4} \sum_{c=3}^{d} \lambda_{c} \Delta_{c}=\frac{N^{2}}{64 \pi^{3}} \sum_{c=3}^{d} \sum_{b=2}^{c}\left(v_{b}, v_{c}, b\right) \Delta_{b} \Delta_{c}=\frac{N^{2} b_{1}}{128 \pi^{3}} \sum_{c=3}^{d} \sum_{b=2}^{c} \sum_{a=1}^{d}\left(v_{b}, v_{c}, v_{a}\right) \Delta_{a} \Delta_{b} \Delta_{c} \\
& =\frac{N^{2} b_{1}}{128 \pi^{3}} \sum_{a=1}^{d} \sum_{b, c \in[2, d]}\left(v_{a}, v_{b}, v_{c}\right) \Delta_{a} \Delta_{b} \Delta_{c}=\frac{N^{2} b_{1}}{128 \pi^{3}} \sum_{1 \leq a<b<c \leq d}\left(v_{a}, v_{b}, v_{c}\right) \Delta_{a} \Delta_{b} \Delta_{c}
\end{aligned}
$$

where we used (B.17) and $b=\frac{b_{1}}{2} \sum_{a} v_{a} \Delta_{a} \cdot{ }^{16}$ Comparing with (2.8), we see that

$$
\mathcal{V}_{\text {on-shell }}\left(b_{i}, \mathfrak{n}_{a}\right)=\left.\frac{b_{1}}{108 \pi^{3}} a\left(\Delta_{a}\right)\right|_{\Delta_{a}=\Delta_{a}(b, \mathfrak{n})} .
$$

Open Access. This article is distributed under the terms of the Creative Commons Attribution License (CC-BY 4.0), which permits any use, distribution and reproduction in any medium, provided the original author(s) and source are credited.

\section{References}

[1] K.A. Intriligator and B. Wecht, The Exact superconformal $R$ symmetry maximizes a, Nucl. Phys. B 667 (2003) 183 [hep-th/0304128] [INSPIRE].

[2] F. Benini and N. Bobev, Exact two-dimensional superconformal R-symmetry and c-extremization, Phys. Rev. Lett. 110 (2013) 061601 [arXiv:1211.4030] [INSPIRE].

[3] F. Benini and N. Bobev, Two-dimensional SCFTs from wrapped branes and c-extremization, JHEP 06 (2013) 005 [arXiv: 1302.4451] [INSPIRE].

[4] D. Martelli, J. Sparks and S.-T. Yau, The Geometric dual of a-maximisation for Toric Sasaki-Einstein manifolds, Commun. Math. Phys. 268 (2006) 39 [hep-th/0503183] [INSPIRE].

[5] D. Martelli, J. Sparks and S.-T. Yau, Sasaki-Einstein manifolds and volume minimisation, Commun. Math. Phys. 280 (2008) 611 [hep-th/0603021] [INSPIRE].

\footnotetext{
${ }^{16}$ In the last step of the proof we reorganized the sum by noticing that the term $\left(v_{1}, v_{e}, v_{f}\right) \Delta_{1} \Delta_{e} \Delta_{f}$ with $2 \leq e<f \leq d$ appears just once while $\left(v_{d}, v_{e}, v_{f}\right) \Delta_{d} \Delta_{e} \Delta_{f}$ with $2 \leq d<e<f \leq d$ appears three times, two with the sign plus and one with sign minus.
} 
[6] Y. Tachikawa, Five-dimensional supergravity dual of a-maximization, Nucl. Phys. B 733 (2006) 188 [hep-th/0507057] [INSPIRE].

[7] P. Szepietowski, Comments on a-maximization from gauged supergravity, JHEP 12 (2012) 018 [arXiv: 1209.3025] [inSPIRE].

[8] P. Karndumri and E. O Colgain, Supergravity dual of c-extremization, Phys. Rev. D 87 (2013) 101902 [arXiv:1302.6532] [INSPIRE].

[9] A. Butti and A. Zaffaroni, R-charges from toric diagrams and the equivalence of a-maximization and Z-minimization, JHEP 11 (2005) 019 [hep-th/0506232] [INSPIRE].

[10] S. Lee and S.-J. Rey, Comments on anomalies and charges of toric-quiver duals, JHEP 03 (2006) 068 [hep-th/0601223] [INSPIRE].

[11] R. Eager, Equivalence of A-Maximization and Volume Minimization, JHEP 01 (2014) 089 [arXiv: 1011.1809] [INSPIRE].

[12] C. Couzens, J.P. Gauntlett, D. Martelli and J. Sparks, A geometric dual of c-extremization, JHEP 01 (2019) 212 [arXiv: 1810.11026] [INSPIRE].

[13] J.P. Gauntlett, D. Martelli and J. Sparks, Toric geometry and the dual of c-extremization, JHEP 01 (2019) 204 [arXiv: 1812.05597] [INSPIRE].

[14] A. Hanany and K.D. Kennaway, Dimer models and toric diagrams, hep-th/0503149 [INSPIRE].

[15] S. Franco, A. Hanany, K.D. Kennaway, D. Vegh and B. Wecht, Brane dimers and quiver gauge theories, JHEP 01 (2006) 096 [hep-th/0504110] [INSPIRE].

[16] B. Feng, Y.-H. He, K.D. Kennaway and C. Vafa, Dimer models from mirror symmetry and quivering amoebae, Adv. Theor. Math. Phys. 12 (2008) 489 [hep-th/0511287] [INSPIRE].

[17] S.M. Hosseini, A. Nedelin and A. Zaffaroni, The Cardy limit of the topologically twisted index and black strings in $A d S_{5}, J H E P 04$ (2017) 014 [arXiv:1611.09374] [INSPIRE].

[18] C.P. Herzog, I.R. Klebanov, S.S. Pufu and T. Tesileanu, Multi-Matrix Models and Tri-Sasaki Einstein Spaces, Phys. Rev. D 83 (2011) 046001 [arXiv:1011.5487] [INSPIRE].

[19] D.L. Jafferis, I.R. Klebanov, S.S. Pufu and B.R. Safdi, Towards the F-Theorem: $N=2$ Field Theories on the Three-Sphere, JHEP 06 (2011) 102 [arXiv:1103.1181] [INSPIRE].

[20] S.M. Hosseini and A. Zaffaroni, Large $N$ matrix models for $3 d \mathcal{N}=2$ theories: twisted index, free energy and black holes, JHEP 08 (2016) 064 [arXiv: 1604.03122] [INSPIRE].

[21] F. Benini, K. Hristov and A. Zaffaroni, Black hole microstates in AdS 4 from supersymmetric localization, JHEP 05 (2016) 054 [arXiv: 1511.04085] [INSPIRE].

[22] F. Benini, K. Hristov and A. Zaffaroni, Exact microstate counting for dyonic black holes in AdS4, Phys. Lett. B 771 (2017) 462 [arXiv:1608.07294] [InSPIRE].

[23] S.M. Hosseini and N. Mekareeya, Large $N$ topologically twisted index: necklace quivers, dualities and Sasaki-Einstein spaces, JHEP 08 (2016) 089 [arXiv: 1604.03397] [INSPIRE].

[24] F. Azzurli, N. Bobev, P.M. Crichigno, V.S. Min and A. Zaffaroni, A universal counting of black hole microstates in $A d S_{4}, J H E P 02$ (2018) 054 [arXiv:1707.04257] [INSPIRE].

[25] I.R. Klebanov and E. Witten, Superconformal field theory on three-branes at a Calabi-Yau singularity, Nucl. Phys. B 536 (1998) 199 [hep-th/9807080] [INSPIRE]. 
[26] B.S. Acharya, J.M. Figueroa-O'Farrill, C.M. Hull and B.J. Spence, Branes at conical singularities and holography, Adv. Theor. Math. Phys. 2 (1999) 1249 [hep-th/9808014] [INSPIRE].

[27] D.R. Morrison and M.R. Plesser, Nonspherical horizons. 1., Adv. Theor. Math. Phys. 3 (1999) 1 [hep-th/9810201] [INSPIRE].

[28] J.P. Gauntlett, D. Martelli, J. Sparks and D. Waldram, Sasaki-Einstein metrics on $S^{2} \times S^{3}$, Adv. Theor. Math. Phys. 8 (2004) 711 [hep-th/0403002] [INSPIRE].

[29] J.P. Gauntlett, D. Martelli, J.F. Sparks and D. Waldram, A New infinite class of Sasaki-Einstein manifolds, Adv. Theor. Math. Phys. 8 (2004) 987 [hep-th/0403038] [INSPIRE].

[30] M. Cvetič, H. Lü, D.N. Page and C.N. Pope, New Einstein-Sasaki spaces in five and higher dimensions, Phys. Rev. Lett. 95 (2005) 071101 [hep-th/0504225] [INSPIRE].

[31] S. Benvenuti, S. Franco, A. Hanany, D. Martelli and J. Sparks, An Infinite family of superconformal quiver gauge theories with Sasaki-Einstein duals, JHEP 06 (2005) 064 [hep-th/0411264] [INSPIRE].

[32] S. Benvenuti and M. Kruczenski, From Sasaki-Einstein spaces to quivers via BPS geodesics: $L^{p, q \mid r}, J H E P 04$ (2006) 033 [hep-th/0505206] [INSPIRE].

[33] A. Butti, D. Forcella and A. Zaffaroni, The Dual superconformal theory for $L^{p, q, r}$ manifolds, JHEP 09 (2005) 018 [hep-th/0505220] [INSPIRE].

[34] S. Franco, A. Hanany, D. Martelli, J. Sparks, D. Vegh and B. Wecht, Gauge theories from toric geometry and brane tilings, JHEP 01 (2006) 128 [hep-th/0505211] [INSPIRE].

[35] A. Butti and A. Zaffaroni, From toric geometry to quiver gauge theory: The Equivalence of a-maximization and Z-minimization, Fortsch. Phys. 54 (2006) 309 [hep-th/0512240] [INSPIRE].

[36] M. Henningson and K. Skenderis, The Holographic Weyl anomaly, JHEP 07 (1998) 023 [hep-th/9806087] [INSPIRE].

[37] S. Benvenuti, L.A. Pando Zayas and Y. Tachikawa, Triangle anomalies from Einstein manifolds, Adv. Theor. Math. Phys. 10 (2006) 395 [hep-th/0601054] [InSPIRE].

[38] S.S. Gubser and I.R. Klebanov, Baryons and domain walls in an $N=1$ superconformal gauge theory, Phys. Rev. D 58 (1998) 125025 [hep-th/9808075] [InSPIRE].

[39] S.S. Gubser, Einstein manifolds and conformal field theories, Phys. Rev. D 59 (1999) 025006 [hep-th/9807164] [INSPIRE].

[40] A. Amariti, L. Cassia and S. Penati, c-extremization from toric geometry, Nucl. Phys. B 929 (2018) 137 [arXiv: 1706. 07752] [INSPIRE].

[41] S.M. Hosseini, I. Yaakov and A. Zaffaroni, Topologically twisted indices in five dimensions and holography, JHEP 11 (2018) 119 [arXiv:1808.06626] [INSPIRE].

[42] F. Benini, N. Bobev and P.M. Crichigno, Two-dimensional SCFTs from D3-branes, JHEP 07 (2016) 020 [arXiv: 1511.09462] [INSPIRE].

[43] A. Amariti and C. Toldo, Betti multiplets, flows across dimensions and c-extremization, JHEP 07 (2017) 040 [arXiv: 1610.08858] [INSPIRE].

[44] A. Amariti, L. Cassia and S. Penati, Surveying 4d SCFTs twisted on Riemann surfaces, JHEP 06 (2017) 056 [arXiv:1703.08201] [INSPIRE]. 
[45] C. Couzens, D. Martelli and S. Schäfer-Nameki, F-theory and $A d S_{3} / C F T_{2}(2,0), J H E P 06$ (2018) 008 [arXiv: 1712.07631] [INSPIRE].

[46] D. Martelli and J. Sparks, Toric geometry, Sasaki-Einstein manifolds and a new infinite class of AdS/CFT duals, Commun. Math. Phys. 262 (2006) 51 [hep-th/0411238] [INSPIRE].

[47] F. Benini and A. Zaffaroni, A topologically twisted index for three-dimensional supersymmetric theories, JHEP 07 (2015) 127 [arXiv:1504.03698] [INSPIRE].

[48] F. Benini and A. Zaffaroni, Supersymmetric partition functions on Riemann surfaces, Proc. Symp. Pure Math. 96 (2017) 13 [arXiv:1605.06120] [INSPIRE].

[49] C. Closset and H. Kim, Comments on twisted indices in 3d supersymmetric gauge theories, JHEP 08 (2016) 059 [arXiv: 1605.06531] [INSPIRE].

[50] O. Aharony, O. Bergman, D.L. Jafferis and J. Maldacena, $N=6$ superconformal Chern-Simons-matter theories, M2-branes and their gravity duals, JHEP 10 (2008) 091 [arXiv:0806.1218] [INSPIRE].

[51] S.L. Cacciatori and D. Klemm, Supersymmetric AdS 4 black holes and attractors, JHEP 01 (2010) 085 [arXiv:0911.4926] [INSPIRE].

[52] G. Dall'Agata and A. Gnecchi, Flow equations and attractors for black holes in $N=2 \mathrm{U}(1)$ gauged supergravity, JHEP 03 (2011) 037 [arXiv:1012.3756] [INSPIRE].

[53] K. Hristov and S. Vandoren, Static supersymmetric black holes in AdS 4 with spherical symmetry, JHEP 04 (2011) 047 [arXiv: 1012.4314] [INSPIRE].

[54] A. Hanany, D. Vegh and A. Zaffaroni, Brane Tilings and M2 Branes, JHEP 03 (2009) 012 [arXiv:0809.1440] [INSPIRE].

[55] L.F. Alday, F. Benini and Y. Tachikawa, Liouville/Toda central charges from M5-branes, Phys. Rev. Lett. 105 (2010) 141601 [arXiv:0909.4776] [InSPIRE].

[56] I. Bah, C. Beem, N. Bobev and B. Wecht, AdS/CFT Dual Pairs from M5-Branes on Riemann Surfaces, Phys. Rev. D 85 (2012) 121901 [arXiv:1112.5487] [INSPIRE].

[57] I. Bah, C. Beem, N. Bobev and B. Wecht, Four-Dimensional SCFTs from M5-Branes, JHEP 06 (2012) 005 [arXiv: 1203.0303] [INSPIRE]. 